\title{
Reflection and transmission of elastic waves at the interface between two gradient-elastic solids with surface energy
}

\author{
Yueqiu Li a , P.J. Wei ${ }^{\text {a, b, *, }}{ }^{\text {Q Qihen Tang }}{ }^{\mathrm{b}}$ \\ a Department of Applied Mechanics, University of Sciences and Technology Beijing, Beijing, 100083, China \\ b State Key Laboratory of Nonlinear Mechanics (LNM), Chinese Academy of Science, Beijing
}

\section{A R T I C L E I N F O}

\section{Article history:}

Received 11 July 2014

Accepted 9 February 2015

Available online 18 February 2015

\section{Keywords:}

Gradient elasticity

Microstructure effects

Surface effects

\begin{abstract}
A B S T R A C T
The reflection and transmission of an incident plane wave at an interface between two gradient elastic solids with surface energy are studied. First, some functions of strain energy density with the surface effects taken into consideration are postulated and the surface effects of gradient elastic solid are incorporated into the constitutive relations. Then, the nontraditional interface conditions by requiring the auxiliary monopolar tractions and the auxiliary dipolar tractions continuous across the interface are used to determine the reflection and transmission coefficients. Some numerical results of the reflection and transmission coefficient in terms of energy flux ratio are given for different microstructure parameters and surface parameters. The microstructure effects and the surface energy effects on the reflection and transmission waves are discussed based on the numerical results. It is found that the microstructure effects result in the appearance of two surface waves and the surface energy effects have apparent different influence on the body waves in gradient elastic solids and the surface waves at the interface. However, this phenomenon becomes pronounced only when the incident wavelength is close to the characteristic length of microstructure.
\end{abstract}

๑ 2015 Elsevier Masson SAS. All rights reserved.

\section{Introduction}

It is known that the propagation of elastic waves in the classical elastic solids is not dispersive and the lattice dynamics shows that the lattice waves are dispersive. The inconsistency between them leads to the requirement of modifying the classical elastic theory when it is used to study the propagation of elastic waves with high frequency or short wavelength. The concept of structural level of deformation more and more becomes recognized recently. According to this concept, each mass point of a continuum is regarded as a complex system of interacting structures at a lower structural level. The elastic theory of solids with microstructure in such hypotheses occupies an intermediate position between the classical continuum theory and the lattice theory. In order to take the microstructure effects into consideration, the generalized elastic theories, for example, the nonlocal theory (Eringen, 2001), the micromorphic theory (Eringen and Gortler, 1964), the couple stress theory (Mindlin and Tiersten, 1962; Toupin, 1962), the micropolar

\footnotetext{
* Corresponding author. Department of Applied Mechanics, University of Sciences and Technology Beijing, Beijing, 100083, China.

E-mail address: weipj@ustb.edu.cn (P.J. Wei).
}

theory (Eringen, 1966) and microstretch theory (Eringen, 1990), were proposed successively in the past several decades. Because the degree of freedom of the material particle increases in these generalized continuum theories, the modes of vibration of material particle become more complicated and therefore create many new wave modes which cannot be observed in the classical elastic solids. Graff and Pao (1967) pointed that there are two propagating body waves (one is dispersive and other is not dispersive) and one dispersive non-propagating body wave in the couple-stress solids. Gourgiotis et al. (2013) pointed that there are two dispersive propagating body waves and two evanescent body waves in the gradient elastic solids. Parfitt and Eringen (1969), Tomar and Gogna (1995) and Tomar and Garg (2005) showed that there are four dispersive body waves in a micropolar solid and five dispersive body waves in the microstretch solids. The microstructure effects not only lead to the new body wave modes in the interior of solid but also the P-type and S-type surface waves at the boundary of solids. It is known that the surface of solid exhibits properties quite different from those associated with their interior. The surface stress is present in liquid in the form of surface tension and more generally may be present at boundary of any solids. Shenoy (2005) studied the surface stress of solids by the atomic simulations and 
demonstrated that the elastic constants of surface can be either positive or negative. Due to the development of nanoscience and nanotechnology, there is an increasing demand for understanding the mechanical behaviours of small-sized materials and structures. At the micro and nano scales, surfaces and interfaces may have significant effects on the mechanical properties of solids due to the increasing ratio of surface/interface area to volume (Duan and et al., 2005; Miller and Shenoy, 2000). Recently, Wang (2007, 2008) studied the diffraction of elastic wave from a nanocylinder and a nanosphere with surface effects. Hasheminejad et al. (Hasheminejad and Avazmohammadi, 2009) and Qiang and Wei (Qiang et al., 2012; Qiang and Wei, 2013) studied the effective propagation constants of elastic wave in random nanocomposite material with the surface effects considered. Liu et al. (2012) and Cai and Wei (2013) further studied the surface/interface effects on band gap of elastic waves in the periodic nanocomposite material. However, in their studies, the surface/interface effects are taken into consideration based on the surface elastic theory proposed by Gurtin and Murdoch (1975). In this surface elastic theory, a surface is regarded as a negligibly thin membrane adhered to the bulk without slipping. The elastic moduli of the thin membrane are different from those of the bulk material. The surface effects are due to the presence of the surface stress in the thin membrane. Ogden and Steigmann (2002) further generalized this theory and proposed that the thin membrane (a coat on the bulk material itself) may have its own bending stiffness. According to this theory, they studied the secular equation of surface wave propagation on a prestressed incompressible isotropic elastic half-space with a thin coating on its plane boundary. Recently, a surface model taking the surface mass, surface elasticity and surface inertia into consideration is proposed by Placidi et al. (2013) and dell'Isola et al. (2012). The surface model was used to model an interface between two second gradient elastic solids and lead to four types of connections between the two sides of the interface, namely, the "generalized internal clamp", the "generalized internal hinge", the "generalized internal roller" and the "generalized internal free ends". All these constraints can be assumed to model different types of surface microstructure which results in different types of connection between the two parts of the continuum.

There is an alternative method to consider the surface effects. The concept of a thin membrane (with elasticity, and mass even) without thickness adhered to the bulk material is discarded. The surface effects are incorporated into the constitutive relation of bulk material by the direct postulation of a specific function of strain energy density, for example, Vardoulakis and Georgiadis (1997), Exadaktylos (1999), Tsepoura et al. (2002), Georgiadis et al. (2000), Yerofeyev and Sheshenina (2005) and others. Recently, the propagation of different types of elastic waves in a gradient-elastic medium with surface energy is studied (Vardoulakis and Georgiadis, 1997; Georgiadis et al., 2000; Yerofeyev and Sheshenina, 2005). The dispersion characteristics of longitudinal and shear body waves, Rayleigh surface waves and antiplane shear surface waves, antiplane shear waves in a layer, and torsional surface waves are analysed. However, the surface effects on the reflection and transmission waves at an interface have not reported up to now. In this paper, the reflection and transmission problems of an incident plane wave at an interface between two gradient elastic solids are studied. The surface effects of dipolar gradient elastic solids are considered by the direct postulation of a specific function of strain energy density. The reflection and transmission coefficients in terms of energy flux ratio are calculated and are validated by the energy conservation through a unit area at interface. The microstructure effects and the surface effects on the reflection and transmission waves are discussed based on the numerical results.

\section{Some specific functions of strain energy density with surface energy}

According to the Mindlin's elastic theory of solids with microstructure, the strain energy density can be expressed as

$$
\begin{aligned}
W= & \frac{1}{2} c_{i j k l} \varepsilon_{i j} \varepsilon_{k l}+\frac{1}{2} b_{i j k l} \gamma_{i j} \gamma_{k l}+\frac{1}{2} a_{i j k l m n} \chi_{i j k} \chi_{l m n}+d_{i j k l m} \gamma_{i j} \chi_{k l m} \\
& +f_{i j k l m} \chi_{i j k} \varepsilon_{l m}+g_{i j k l} \gamma_{i j} \varepsilon_{k l},
\end{aligned}
$$

where $c_{i j k l}, b_{i j k l}, a_{i j k l m n}, d_{i j k l m} f_{i j k l m}$ and $g_{i j k l}$ are the components of elastic tensors of materials. $\varepsilon_{i j}, \gamma_{i j}\left(=u_{j, i}-\psi_{i j}\right)$ and $\chi_{i j k}\left(=\psi_{j k, i}\right)$ are the macro-strain of macro-medium, relative deformation (the difference between the macro-displacement gradient and the microdeformation) and the micro-deformation gradient (the macrogradient of the micro-deformation), respectively. If the relative deformation is assumed to be zero, namely, $\gamma_{i j}=0$, then, the strain energy density function is simplified as

$W=\frac{1}{2} c_{i j k l} \varepsilon_{i j} \varepsilon_{k l}+\frac{1}{2} a_{i j k l m n} \chi_{i j k} \chi_{l m n}+f_{i j k l m} \chi_{i j k} \varepsilon_{l m}$,

It means that the strain energy density is not only dependent of the strain but also the strain gradient. Even for isotropic medium, there are too many material parameters involved still. Here, a simplified version of Eq. (2) is given as following.

$$
\begin{aligned}
W= & \left(\frac{1}{2} \lambda \varepsilon_{i i} \varepsilon_{j j}+\mu \varepsilon_{i j} \varepsilon_{i j}\right)+\left(\frac{1}{2} \lambda c \varepsilon_{i i, k} \varepsilon_{j j, k}+\mu c \varepsilon_{i j, k} \varepsilon_{j i, k}\right) \\
& +\left[\frac{1}{2} \lambda b_{k}\left(\varepsilon_{i i} \varepsilon_{j j}\right)_{, k}+\mu b_{k}\left(\varepsilon_{i j} \varepsilon_{j i}\right)_{, k}\right]
\end{aligned}
$$

where the first term is the contribution from the strains in bulk material; the second term is the contribution from the strain gradient in bulk material. Consider that

$\int_{V} b_{k}\left(\varepsilon_{i j} \varepsilon_{j i}\right)_{, k} \mathrm{~d} V=\int_{S}\left(\varepsilon_{i j} \varepsilon_{j i}\right) b_{k} n_{k} \mathrm{~d} S$,

The third term in Eq. (3) is the contribution from the surface material. Let

$E_{1}=\frac{1}{2} \lambda b_{k} n_{k}\left(\varepsilon_{i i} \varepsilon_{j j}\right)+\mu b_{k} n_{k}\left(\varepsilon_{i j} \varepsilon_{j i}\right)$

then, $E_{1}$ is the surface energy of unit surface area and it includes the contribution from the surface normal stress and the surface shear stress, respectively. Define

$\tau_{i j}=\frac{\partial W}{\partial \varepsilon_{i j}}=\left(\lambda \delta_{i j} \varepsilon_{p p}+2 \mu \varepsilon_{i j}\right)+b_{k}\left(\lambda \delta_{i j} \varepsilon_{p p, k}+2 \mu \varepsilon_{i j, k}\right)$,

$\mu_{k i j}=\frac{\partial W}{\partial \chi_{k i j}}=b_{k}\left(\lambda \delta_{i j} \varepsilon_{p p}+2 \mu \varepsilon_{i j}\right)+c\left(\lambda \delta_{i j} \varepsilon_{p p, k}+2 \mu \varepsilon_{i j, k}\right)$.

where $\lambda$ and $\mu$ are the classical lame constants; $c$ is a microstructure parameter with dimension of $\mathrm{m}^{2} ; b_{k}$ is a surface parameters with dimension of m. $\tau_{i j}$ is the Cauchy stress or monopolar stress with the dimensions of $\mathrm{Nm}^{-2}$ and $\mu_{i j k}$ the dipolar stress with the dimensions of $\mathrm{Nm}^{-1}$. The monopolar stress and the dipolar stress are corresponding with the notion of monopolar force and the dipolar force, respectively. The monopolar force is the classical force and the dipolar forces are the anti-parallel forces acting between the micro-media contained in the continuum with microstructure. 


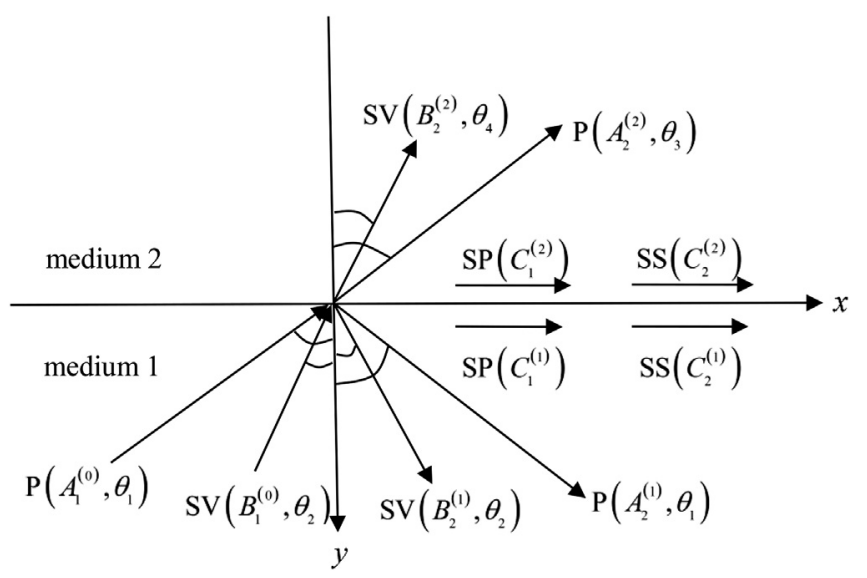

Fig. 1. Reflection and transmission waves at an interface between two different microstructure solids for an oblique incident plane wave.

If the motion of micro-medium involves only the rigid rotation, then,

$W=\mu\left[\varepsilon_{i j} \varepsilon_{i j}+c \varepsilon_{i j, k} \varepsilon_{j i, k}+b_{k}\left(\varepsilon_{i j} \varepsilon_{i j}\right)_{, k}\right]$,

$\tau_{i j}=2 \mu\left(\varepsilon_{i j}+b_{k} \varepsilon_{i j, k}\right)$, $\mu_{k i j}=2 \mu\left(b_{k} \varepsilon_{i j}+c \varepsilon_{i j, k}\right)$,

If the motion of micro-medium is irrotational, then,

$W=\frac{1}{2} \lambda\left[\varepsilon_{i i} \varepsilon_{j j}+c \varepsilon_{i i, k} \varepsilon_{j j, k}+b_{k}\left(\varepsilon_{i i} \varepsilon_{j j}\right)_{, k}\right]$

$\tau_{i j}=\lambda \delta_{i j}\left(\varepsilon_{p p}+b_{k} \varepsilon_{p p, k}\right)$,

$\mu_{k i j}=\lambda \delta_{i j}\left(b_{k} \varepsilon_{p p}+c \varepsilon_{p p, k}\right)$.

The kinetic energy density includes two terms. One involves the velocity, and the other involves the velocity gradient

$T=\frac{1}{2} \rho \dot{u}_{j} \dot{u}_{j}+\frac{1}{6} \rho d^{2} \dot{u}_{k, j} \dot{u}_{k, j}$

where, $d$ is the characteristic length of microstructure. $\rho$ is the mass density.

The work done by the external forces is

$W_{1}=\int_{V} F_{k} u_{k} \mathrm{~d} V+\int_{S} P_{k} u_{k} \mathrm{~d} S+\int_{S} R_{k} D u_{k} \mathrm{~d} S$,
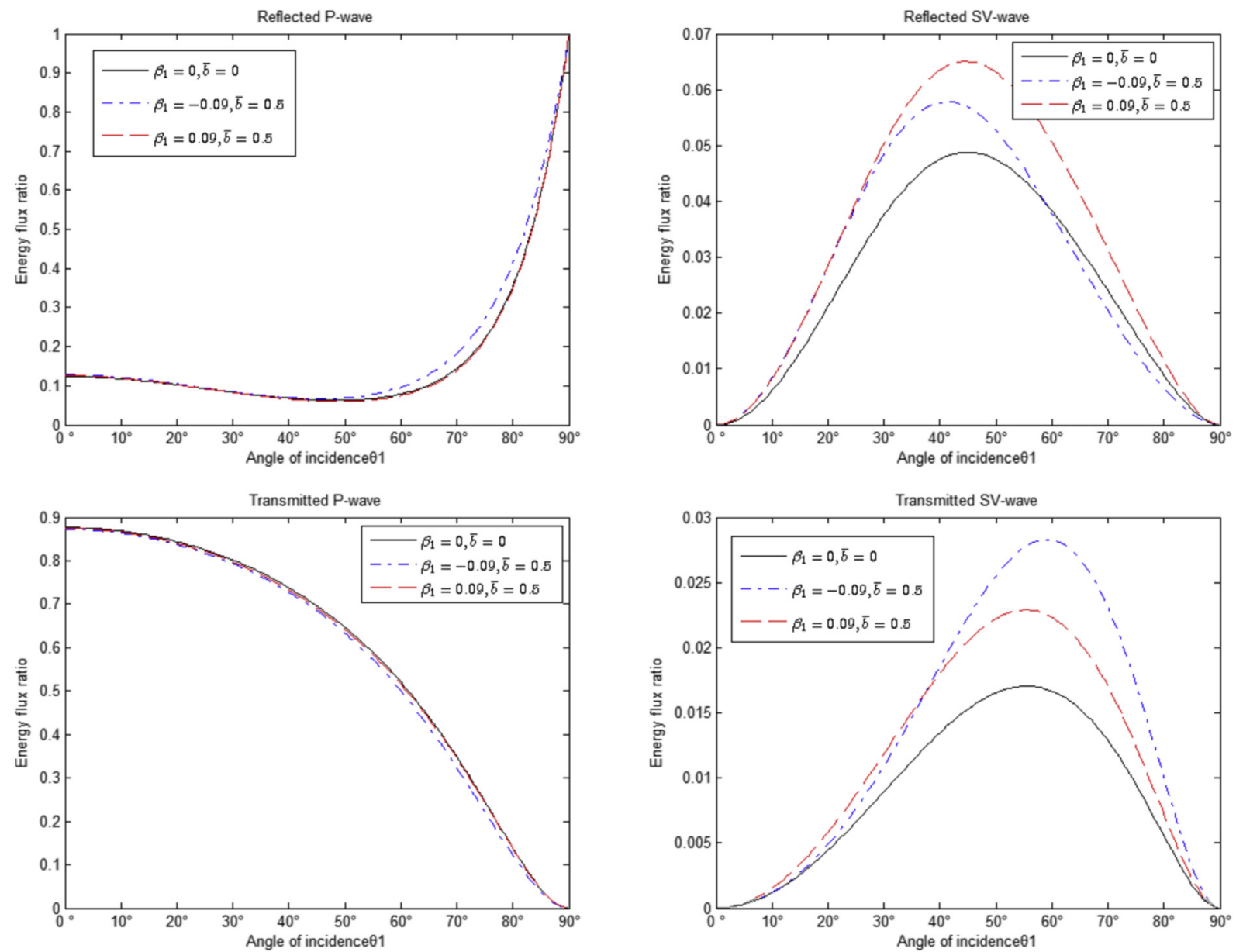

Fig. 2. The reflection and transmission coefficients of body waves under an incident P-wave for different $\beta_{1}\left(\bar{b}=0.5, \quad \alpha_{1}=0.1, \quad \bar{\lambda}=10\right)$. 
where $F_{k}$ is the body force. $P_{k}$ is the monopolar traction. $R_{k}$ is the dipolar tractions. $D u_{k}$ is the normal derivative of displacement component.

By the application of the Hamilton variational principle

$\delta \int_{t_{0}}^{t_{1}} \int_{V}(T-W) d V d t+\int_{t_{0}}^{t_{1}} \int_{S} \delta W_{1} d S d t=0$,

The motion equation and the boundary conditions are obtained as

$\left(\tau_{j k}-\mu_{i j k, i}\right)_{j}+F_{k}=\rho \ddot{u}_{k}-\frac{\rho d^{2}}{3} \ddot{u}_{k, j j}$

$P_{k}=n_{j}\left(\tau_{j k}-\mu_{i j k, i}\right)-D_{j}\left(n_{i} \mu_{i j k}\right)+\left(D_{l} n_{l}\right) n_{i} n_{j} \mu_{i j k}+\frac{\rho d^{2}}{3} n_{j} \ddot{u}_{k, j}$,

$R_{k}=n_{i} n_{j} \mu_{i j k}$

where $n_{j}$ is the normal of the boundary of solid. $D_{j}=\left(\delta_{j l}-n_{j} n_{l}\right) \partial_{l}$, $D=n_{l} \partial_{l}$.
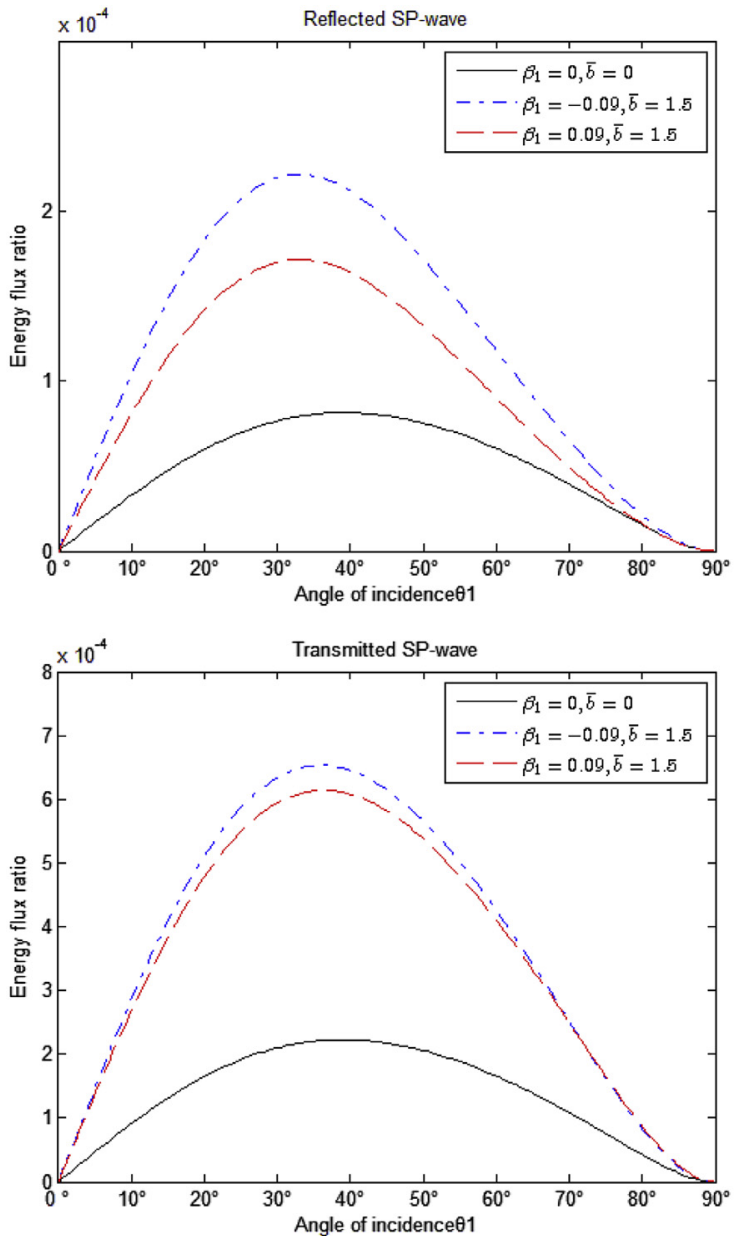

\section{Reflection and transmission at the interface}

Inserting Eqs. (6) and (7) into Eq. (13) and ignoring the volume force leads to the equation of motion in terms of displacement vector.

$\left(1-c \nabla^{2}\right)\left[(\lambda+\mu) \nabla \nabla \cdot \mathbf{u}+\mu \nabla^{2} \mathbf{u}\right]=\rho \ddot{\mathbf{u}}-\frac{\rho d^{2}}{3} \nabla^{2} \ddot{\mathbf{u}}$,

where $\nabla^{2}$ is the Laplace operator. Eq. (16) reduces to Navier equation in the classical elastic theory when the microstructure parameter $c$ and $d$ are taken to be zero. It is noted that the microstructure parameter $c$ and $d$ appear in the equation but the surface parameter $b_{k}$ does not appear in the equation. It means that the microstructure effects will change the wave propagation modes in the solid but the surface effect does not. After taking the divergence and curl operation on both sides of Eq. (16) leads to

$V_{p}^{2}\left(1-c \nabla^{2}\right) \nabla^{2} \nabla \cdot \mathbf{u}=\left(1-\frac{d^{2}}{3} \nabla^{2}\right) \nabla \cdot \ddot{\mathbf{u}}$,

$V_{s}^{2}\left(1-c \nabla^{2}\right) \nabla^{2} \nabla \times \mathbf{u}=\left(1-\frac{d^{2}}{3} \nabla^{2}\right) \nabla \times \ddot{\mathbf{u}}$,

where
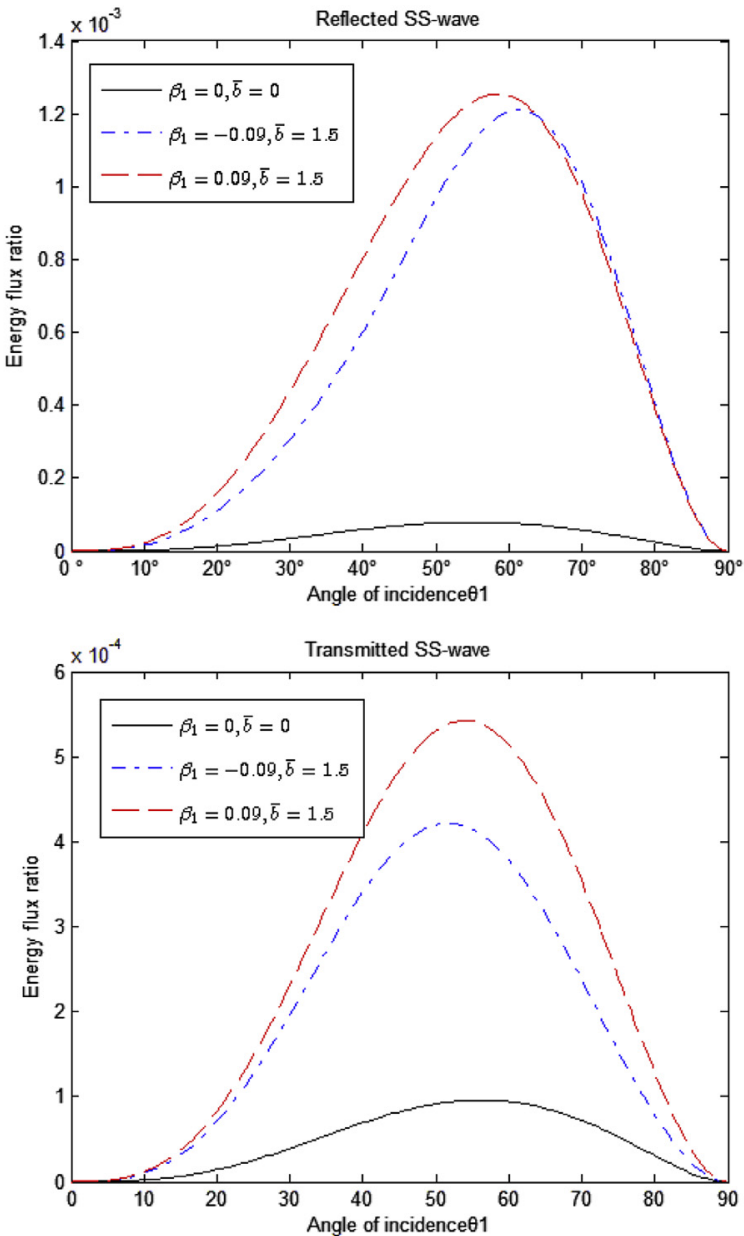

Fig. 3. The reflection and transmission coefficients of surface waves under an incident P-wave for different $\beta_{1}\left(\bar{b}=1.5, \quad \alpha_{1}=0.1, \quad \bar{\lambda}=10\right)$. 
$V_{p}^{2}=\frac{\lambda+2 \mu}{\rho}, \quad V_{s}^{2}=\frac{\mu}{\rho}$.

Assume that the displacement field is of form

$\mathbf{u}=\operatorname{Aexp}[i(\sigma v \cdot \mathbf{r}-\omega t)]$,

Then, the dispersive relation of dilatational and distortional waves can be obtained

$\omega^{2}=\sigma_{p}^{2} V_{p}^{2}\left(1+c \sigma_{p}^{2}\right)\left(1+\frac{d^{2}}{3} \sigma_{p}^{2}\right)^{-1}$,

$\omega^{2}=\sigma_{s}^{2} V_{s}^{2}\left(1+c \sigma_{s}^{2}\right)\left(1+\frac{d^{2}}{3} \sigma_{s}^{2}\right)^{-1}$

where $\sigma_{p}$ and $\sigma_{s}$ are the wave numbers of dilatational and distortional waves, respectively.

In order to uncouple the vector equation (16), the application of Helmholtz vector decomposition

$\mathbf{u}=\nabla \varphi+\nabla \times \psi, \quad \nabla \cdot \psi=0$,

leads to
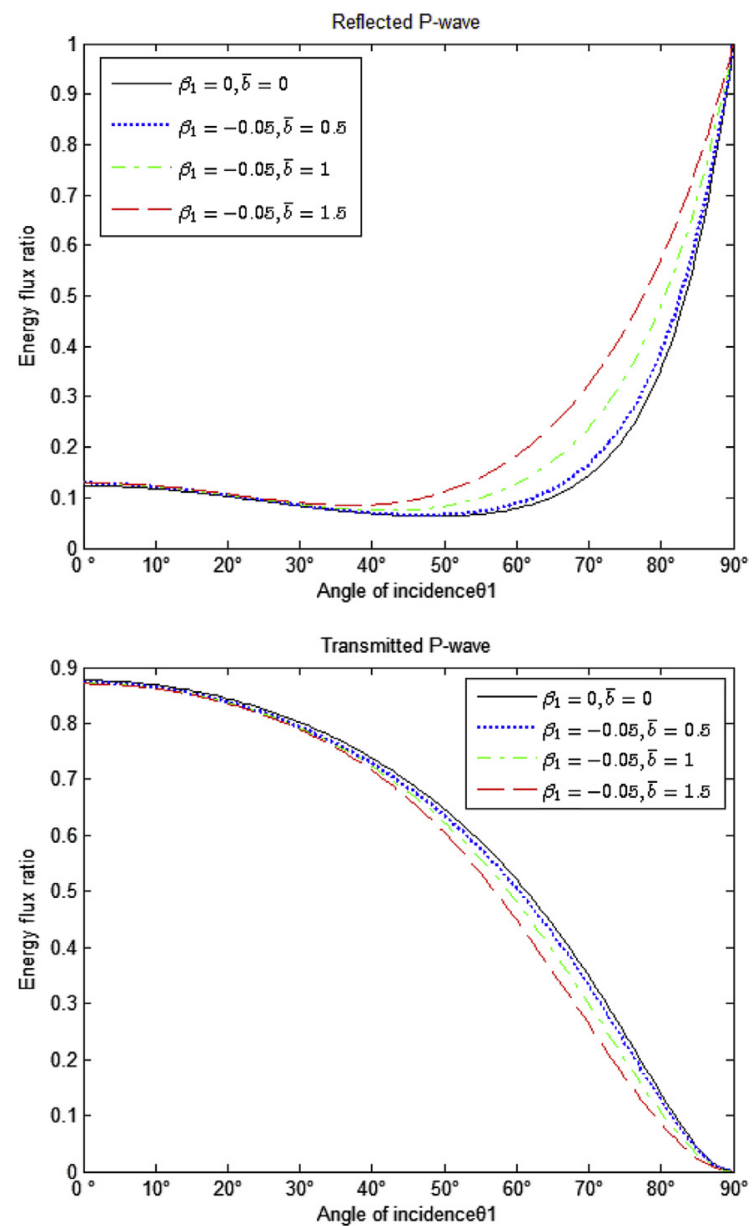

$V_{p}^{2}\left(1-c \nabla^{2}\right) \nabla^{2} \varphi=\left(1-\frac{d^{2}}{3} \nabla^{2}\right) \ddot{\varphi}$

$V_{s}^{2}\left(1-c \nabla^{2}\right) \nabla^{2} \psi=\left(1-\frac{d^{2}}{3} \nabla^{2}\right) \ddot{\psi}$

Eq. (21) can be rewritten as

$\left(\nabla^{2}+\sigma_{p}^{2}\right)\left(\nabla^{2}-\tau_{p}^{2}\right) \varphi=0$

$\left(\nabla^{2}+\sigma_{s}^{2}\right)\left(\nabla^{2}-\tau_{s}^{2}\right) \psi=0$

Eq. (22) means that there are two travelling waves with wave number $\sigma_{p}$ and $\sigma_{s}$ and two evanescent waves with attenuation factor $\tau_{p}$ and $\tau_{s}$. The two evanescent waves reduce to the P-type and S-type surface waves (indicated by SP and SS waves, respectively) when they propagate along the surface. In the reflection and transmission problem, the apparent wave number of all waves (incident waves, reflection waves and transmission waves) should be equal. Let consider the solutions of form

$\varphi(x, y, t)=\tilde{\varphi}(y) \exp [i(\xi x-\omega t)]$

$\psi(x, y, t)=\tilde{\psi}(y) \exp [i(\zeta x-\omega t)]$

Inserting Eq. (23) into Eq. (22) leads to
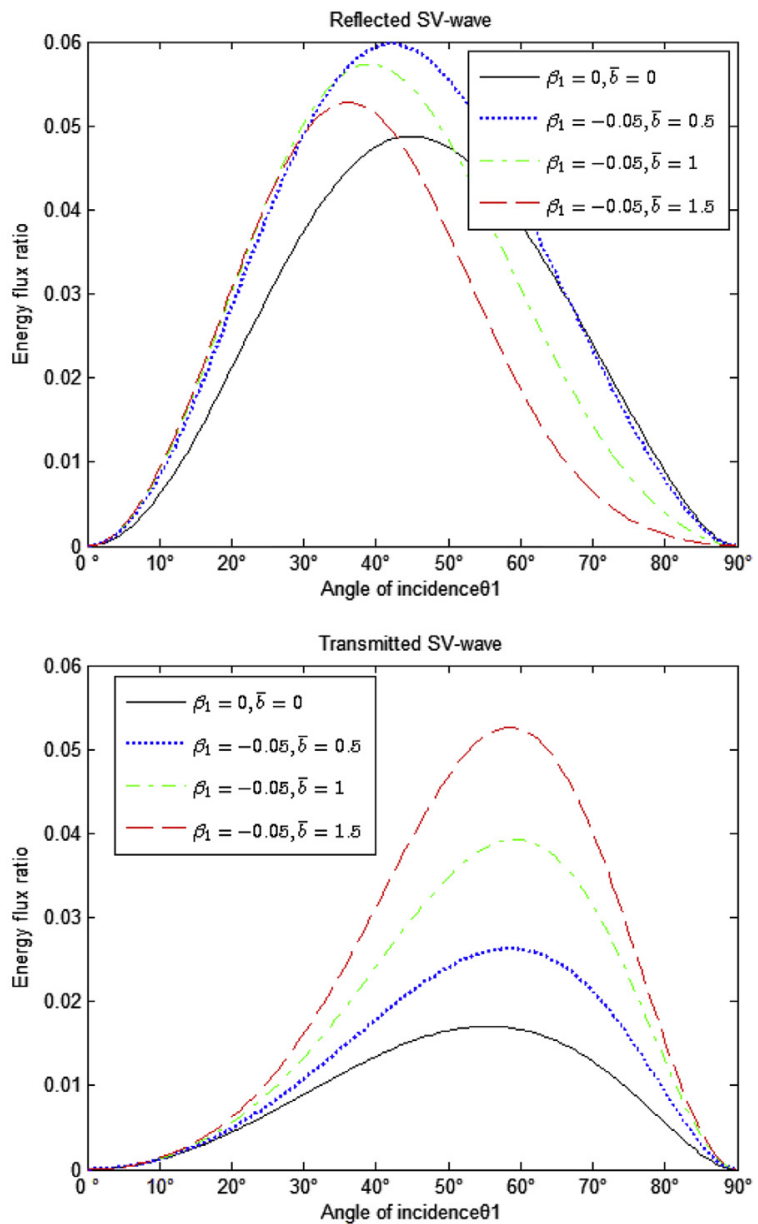

Fig. 4. The reflection and transmission coefficients of body waves under an incident P-wave for different $\left(\bar{b}\left(\beta_{1}=0.05, \quad \alpha_{1}=0.1, \quad \bar{\lambda}=10\right)\right.$. 
$\left(\frac{d^{2}}{d y^{2}}+\beta_{p}^{2}\right)\left(\frac{d^{2}}{d y^{2}}-\gamma_{p}^{2}\right) \tilde{\varphi}(y)=0$

$\left(\frac{d^{2}}{d y^{2}}+\beta_{s}^{2}\right)\left(\frac{d^{2}}{d y^{2}}-\gamma_{s}^{2}\right) \tilde{\psi}(y)=0$,

where

$\beta_{p}^{2}=\sigma_{p}^{2}-\xi^{2}, \quad \gamma_{p}^{2}=\tau_{p}^{2}+\xi^{2}, \quad \beta_{s}^{2}=\sigma_{s}^{2}-\zeta^{2}, \quad \gamma_{s}^{2}=\tau_{s}^{2}+\xi^{2}$,

$\sigma_{p}=\left\{\frac{1}{2 c}\left[\Delta_{p}-\left(1-a_{p}\right)\right]\right\}^{\frac{1}{2}}, \quad \tau_{p}=\left\{\frac{1}{2 c}\left[\Delta_{p}+\left(1-a_{p}\right)\right]\right\}^{\frac{1}{2}}$,

$\sigma_{s}=\left\{\frac{1}{2 c}\left[\Delta_{s}-\left(1-a_{s}\right)\right]\right\}^{\frac{1}{2}}, \quad \tau_{s}=\left\{\frac{1}{2 c}\left[\Delta_{s}+\left(1-a_{s}\right)\right]\right\}^{\frac{1}{2}}$,

$a_{p}=\frac{\omega^{2} d^{2}}{3 V_{p}^{2}}, \quad a_{s}=\frac{\omega^{2} d^{2}}{3 V_{s}^{2}}, \quad \Delta_{p}=\left[\left(1-a_{p}\right)^{2}+\frac{4 c \omega^{2}}{V_{p}^{2}}\right]^{\frac{1}{2}}$

$\Delta_{s}=\left[\left(1-a_{s}\right)^{2}+\frac{4 c \omega^{2}}{V_{s}^{2}}\right]^{\frac{1}{2}}$.

If the strain energy function (8a) is used instead of (3), then, the wave propagation modes do not change. Only the following two parameters used to calculate the wavenumbers of $\mathrm{P}$ wave and SP wave need to be modified as

$a_{p}=\frac{\omega^{2} d^{2}}{6 V_{s}^{2}}, \quad \Delta_{p}=\left[\left(1-a_{p}\right)^{2}+\frac{2 c \omega^{2}}{V_{s}^{2}}\right]^{\frac{1}{2}}$

If the strain energy function (9a) is used instead of (3), then, SV body wave and SS surface wave will disappear and the following two parameters used to calculate the wavenumbers of $P$ wave and SP wave need to be modified as

$a_{p}=\frac{\omega^{2} d^{2}}{3\left(V_{p}^{2}-2 V_{s}^{2}\right)}, \quad \Delta_{p}=\left[\left(1-a_{p}\right)^{2}+\frac{4 c \omega^{2}}{V_{p}^{2}-2 V_{s}^{2}}\right]^{\frac{1}{2}}$

It is noted that both $\gamma_{p}^{2}$ and $\gamma_{s}^{2}$ always are greater than zero. Whereas $\beta_{p}^{2}$ and $\beta_{s}^{2}$ may have real values greater than, equal to, or less than zero. When both $\beta_{p}^{2}$ and $\beta_{s}^{2}$ are greater than zero, which represents the situation that both dilatational and transverse propagating waves exist, the solution of Eq. (23) can be expressed as

$$
\begin{aligned}
\varphi= & A_{1} \exp \left[i\left(\xi x-\beta_{p} y-\omega t\right)\right]+A_{2} \exp \left[i\left(\xi x+\beta_{p} y-\omega t\right)\right] \\
& +C_{1} \exp \left[+\gamma_{p} y+i(\xi x-\omega t)\right]+C_{2} \exp \left[-\gamma_{p} y\right. \\
& +i(\xi x-\omega t)]
\end{aligned}
$$
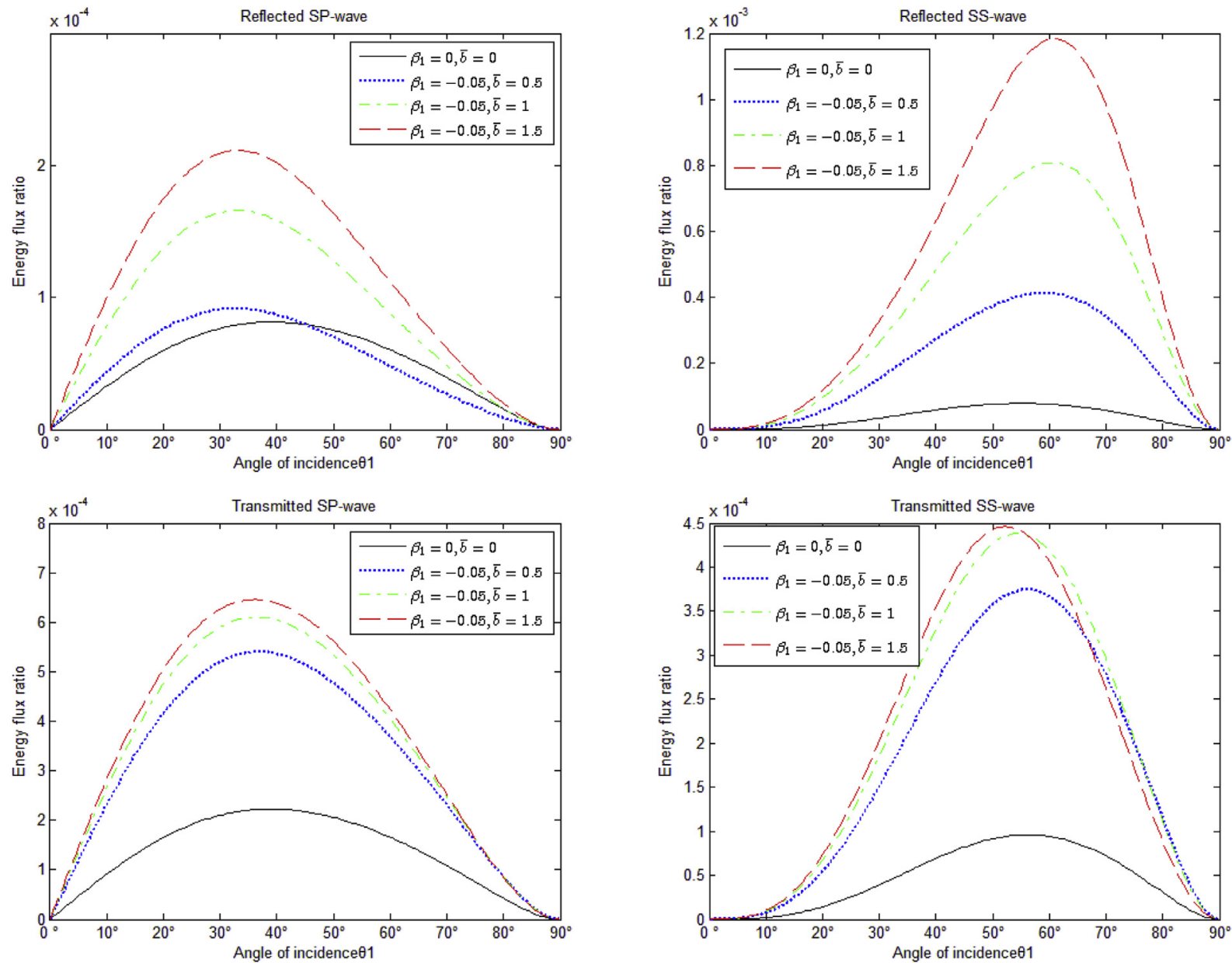

Fig. 5. The reflection and transmission coefficients of surface waves under an incident P-wave for different $\bar{b}\left(\beta_{1}=-0.05, \alpha_{1}=0.1, \bar{\lambda}=10\right)$. 


$$
\begin{aligned}
\psi= & B_{1} \exp \left[i\left(\zeta x-\beta_{s} y-\omega t\right)\right]+B_{2} \exp \left[i\left(\zeta x+\beta_{s} y-\omega t\right)\right]+C_{3} \exp [ \\
& \left.+\gamma_{s} y+i(\zeta x-\omega t)\right]+C_{4} \exp \left[-\gamma_{s} y+i(\zeta x-\omega t)\right]
\end{aligned}
$$

Consider an incident plane $\mathrm{P}$ wave from medium 1 propagates obliquely toward the interface, see Fig. 1 . When it impinges the interface, the reflection waves in medium 1 and the transmission waves in medium 2 are created. Let $A_{1}^{(i)}, A_{2}^{(i)}, B_{1}^{(i)}, B_{2}^{(i)}, C_{1}^{(i)}, C_{2}^{(i)}$ represents the amplitude of each waves in microstructure solids $(i=1$ for medium 1 and $i=2$ for medium $2, i=0$ represents the incident waves), respectively. Define $A_{2}^{(1)} / A_{1}^{(0)}, B_{2}^{(1)} / A_{1}^{(0)}, C_{1}^{(1)} / A_{1}^{(0)}$ and $C_{2}^{(1)} / A_{1}^{(0)}$ be the amplitude ratio of various reflection waves (dilatational wave, distortional wave, P-type surface wave and the S-type surface wave) with respect to that of incident wave. Similarly, $A_{2}^{(2)} / A_{1}^{(0)}, B_{2}^{(2)} / A_{1}^{(0)}$, $C_{1}^{(2)} / A_{1}^{(0)}$ and $C_{2}^{(2)} / A_{2}^{(0)}$ are the amplitude ratio of various transmission waves with respect to the incident wave. These amplitude ratios are determined by the interface conditions between two microstructured solids. For the dipolar gradient elastic solids considered in this paper, the interface conditions can be expressed as

$\left.\left(u_{i}^{(1)}-u_{i}^{(2)}\right)\right|_{y=0}=0, \quad(i=x, y)$,

$\left.\left(n_{y} u_{i, y}^{(1)}-n_{y} u_{i, y}^{(2)}\right)\right|_{y=0}=0$

$$
\left.\left(P_{i}^{(1)}-P_{i}^{(2)}\right)\right|_{y=0}=0
$$

$$
\left.\left(R_{i}^{(1)}-R_{i}^{(2)}\right)\right|_{y=0}=0
$$

where

$$
\begin{aligned}
P_{x}= & 2 \mu\left(1-c \nabla^{2}\right) \varepsilon_{y x}-\left(c \frac{\partial}{\partial y}+b_{y}\right)\left[(\lambda+2 \mu) \varepsilon_{x x, x}+\lambda \varepsilon_{y y, x}\right] \\
& +\frac{\rho d^{2}}{3} \ddot{u}_{x, y}
\end{aligned}
$$

$$
\begin{aligned}
P_{y}= & \left(1-c \nabla^{2}\right)\left[(\lambda+2 \mu) \varepsilon_{y y}+\lambda \varepsilon_{x x}\right]-2 \mu\left(c \frac{\partial}{\partial y}+b_{y}\right) \varepsilon_{x y, x} \\
& +\frac{\rho d^{2}}{3} \ddot{u}_{y, y}
\end{aligned}
$$

$R_{x}=2 \mu\left(c \frac{\partial}{\partial y}+b_{y}\right) \varepsilon_{y x}$

$R_{y}=\left(c \frac{\partial}{\partial y}+b_{y}\right)\left[(\lambda+2 \mu) \varepsilon_{y y}+\lambda \varepsilon_{x x}\right]$
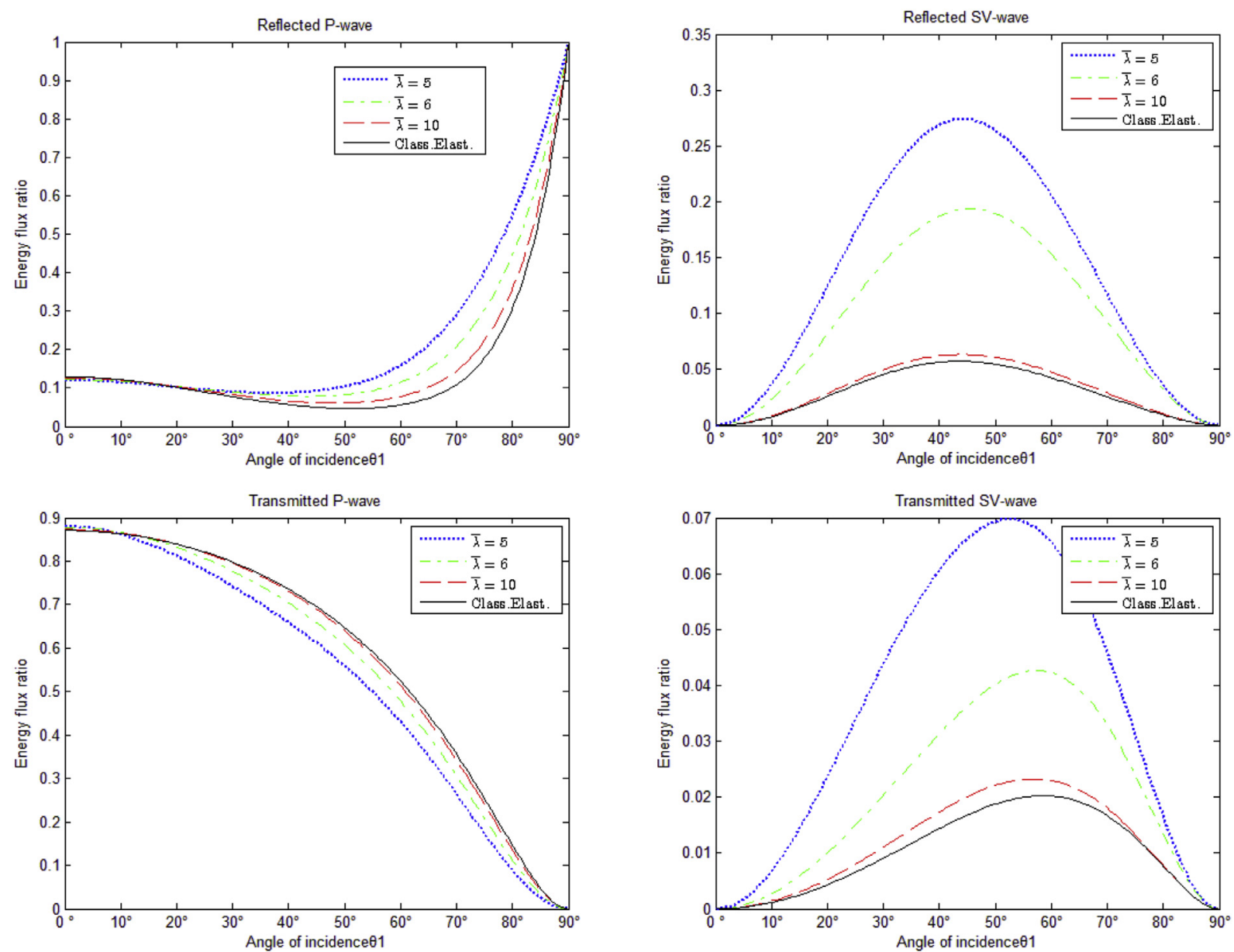

Fig. 6. The reflection and transmission coefficients of body waves under an incident P-wave for different $\bar{\lambda}\left(\beta_{1}=0.05, \bar{b}=0.5, \alpha_{1}=0.1\right)$. 
It is noted that only surface parameter $b_{y}$ in Eq. (3) are included in the explicit expression of monopolar tractions and dipolar tractions when the normal of interface is along $y$ axis. Therefore, the surface parameter $b_{x}$ has no influences on the monopolar tractions and dipolar tractions. The interface conditions mean that the displacement, the normal derivative of displacement, the monopolar traction and the dipolar traction are continuous across interface. It should be remarked that the different surface microstructure may result in the different form of interface conditions. The interface conditions used in this paper is corresponding with the "generalized internal clamp" discussed in (Placidi et al., 2013). Eq. (28) can be written in the matrix form

$\mathbf{A x}=\mathbf{B}+\mathbf{C}$

where

$$
\begin{aligned}
\mathbf{x}= & \left(A_{2}^{(1)} / A_{1}^{(0)}, C_{1}^{(1)} / A_{1}^{(0)}, B_{2}^{(1)} / A_{1}^{(0)}, C_{2}^{(1)} / A_{1}^{(0)}, A_{2}^{(2)} / A_{1}^{(0)}, C_{1}^{(2)}\right. \\
& \left./ A_{1}^{(0)}, B_{2}^{(2)} / A_{1}^{(0)}, C_{2}^{(2)} / A_{1}^{(0)}\right) .
\end{aligned}
$$

The explicit expression of the elements of matrix $\mathbf{A}, \mathbf{B}$ and $\mathbf{C}$ are listed in the appendix A. B is related with the incident dilatational wave and $\mathbf{C}$ with the incident distortional wave. The reflection and transmission amplitude ratios $\mathbf{x}$ can be obtained by solving Eq. (30). It should be pointed that the surface effect does not change the wave propagation mode in microstructured solid although, it change the monopolar traction and the dipolar traction and hence
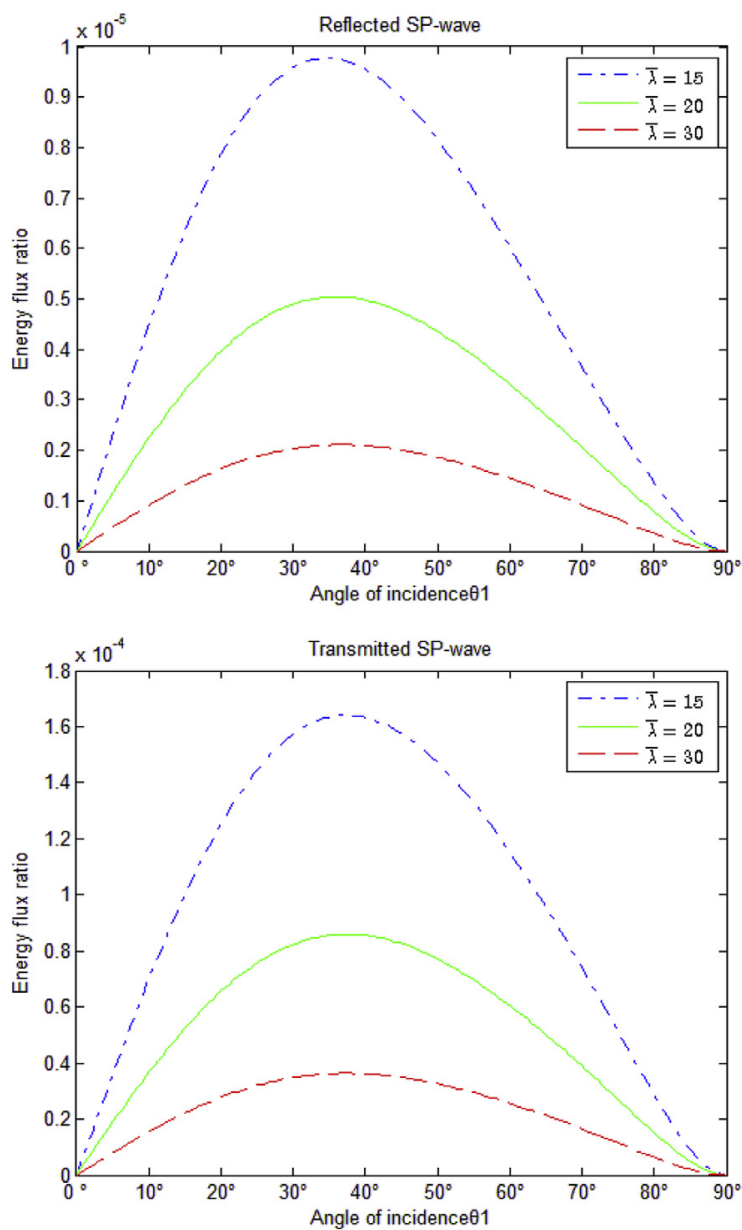

leads to the change of the boundary conditions. As a result, the reflection and transmission amplitude ratios are influenced by the surface effects. If the strain energy density function (9a) is used instead of (3), then, only the P wave and the SP wave exist. Accordingly, the Eq. (28) for $i=x$ in the boundary conditions are not necessary. The matrix A reduces to $4 \times 4$ order from $8 \times 8$ order. In the case of normal incidence, no wave mode conversion and surface wave exist. Then, the matrix A reduces to $2 \times 2$ order. Due to only two unknown coefficients, namely, the reflection and transmission coefficients of body wave, to be determined, only two interface conditions are necessary. The interface conditions Eq. (28) can be divided into two groups: the group A includes Eqs. (28a) and (28c) and the group B includes Eqs. (28b) and (28d). The group A can reduce to the interface conditions of classical elastic solids as the wavelength increases gradually while the group B involves more microstructure informations.

It is known that $\mathbf{x}$ is dependent upon the material constants $\left(\nu_{i}, E_{i}, \rho_{i}, c_{i}, d_{i}, b_{i}\right)$ of two microstructured solids and the parameter of incident wave $\left(A_{1}^{(0)}, \lambda, \omega, \theta\right)$, namely,

$$
\begin{gathered}
\left(A_{j}^{(i)}, B_{j}^{(i)}, C_{j}^{(i)}\right) / A_{1}^{(0)}=f\left(\nu_{1}, E_{1}, \rho_{1}, c_{1}, b_{1 y}, d_{1}, \nu_{2}, E_{2}, \rho_{2}, c_{2},\right. \\
\left.\times b_{2 y}, d_{2}, \lambda, \omega, \theta\right) .
\end{gathered}
$$

Choose $(\rho, d, \omega)$ as the basic physical quantities, then, the nondimensional form of Eq. (31) is
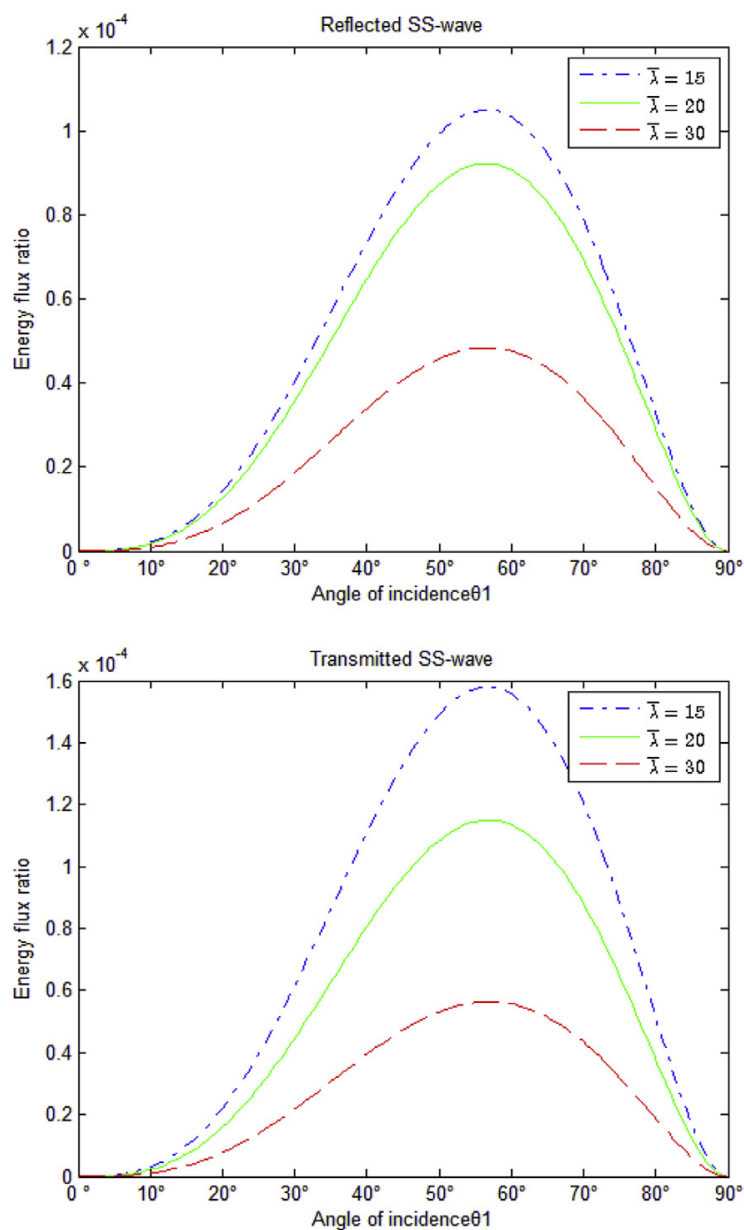

Fig. 7. The reflection and transmission coefficients of surface waves under an incident P-wave for different $\bar{\lambda}\left(\beta_{1}=0.05, \bar{b}=0.5, \alpha_{1}=0.1\right)$. 

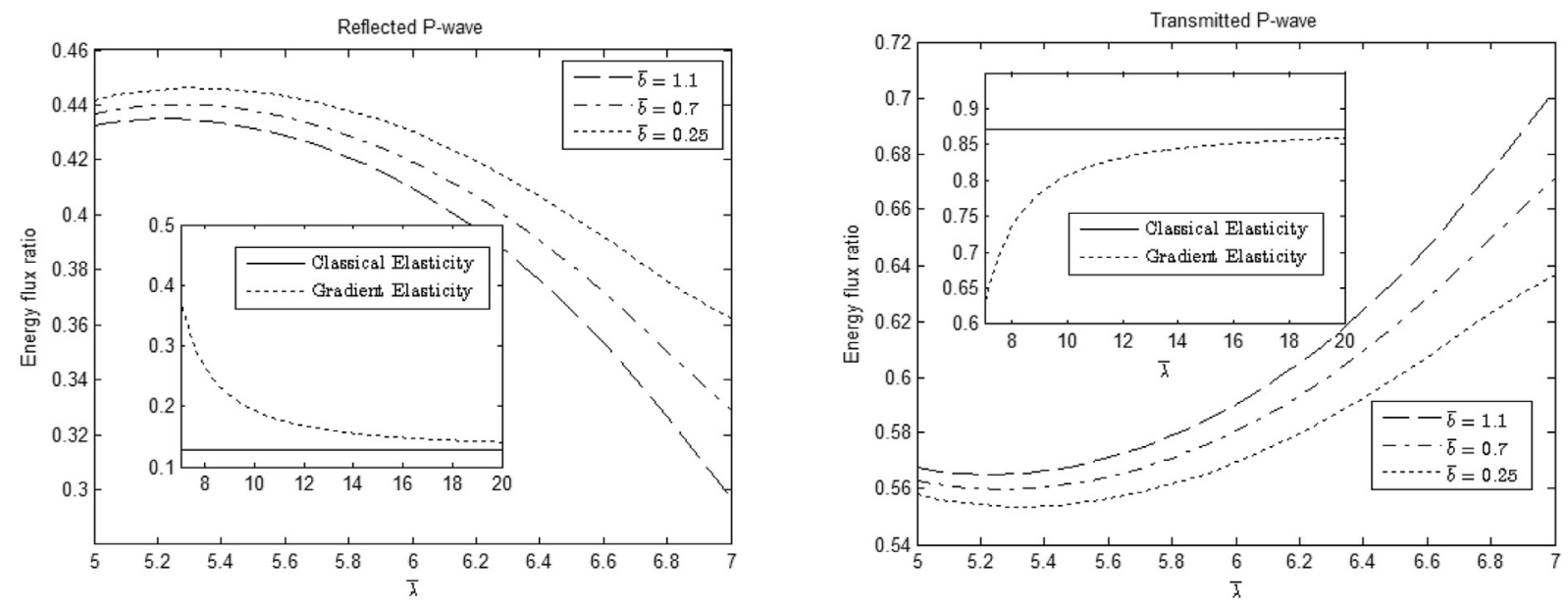

Fig. 8. The reflection and transmission coefficients of body waves under a normal incident P-wave for different $\bar{b}\left(\alpha_{1}=0.05, \beta_{1}=0.005\right)$.

$\left(A_{j}^{(i)}, B_{j}^{(i)}, C_{j}^{(i)}\right) / A_{1}^{(0)}=f\left(\nu_{1}, 1,1, \alpha_{1}, \beta_{1}, 1, \bar{\nu}, \bar{E}, \bar{\rho}, \bar{c}, \bar{b}, \bar{d}, \bar{\lambda}, 1, \theta\right)$

where

$\alpha_{1}=\frac{\sqrt{c_{1}}}{d_{1}}, \quad \beta_{1}=\frac{b_{1 y}}{d_{1}}, \quad \bar{b}=\frac{b_{2 y}}{b_{1 y}}, \quad \bar{d}=\frac{d_{2}}{d_{1}}, \quad \bar{c}=\frac{c_{2}}{c_{1}}$, $\bar{E}=\frac{E_{2}}{E_{1}}, \quad \bar{\nu}=\frac{\nu_{2}}{\nu_{1}}, \quad \bar{\rho}=\frac{\rho_{2}}{\rho_{1}}, \quad \bar{\lambda}=\frac{\lambda}{d_{1}}$.
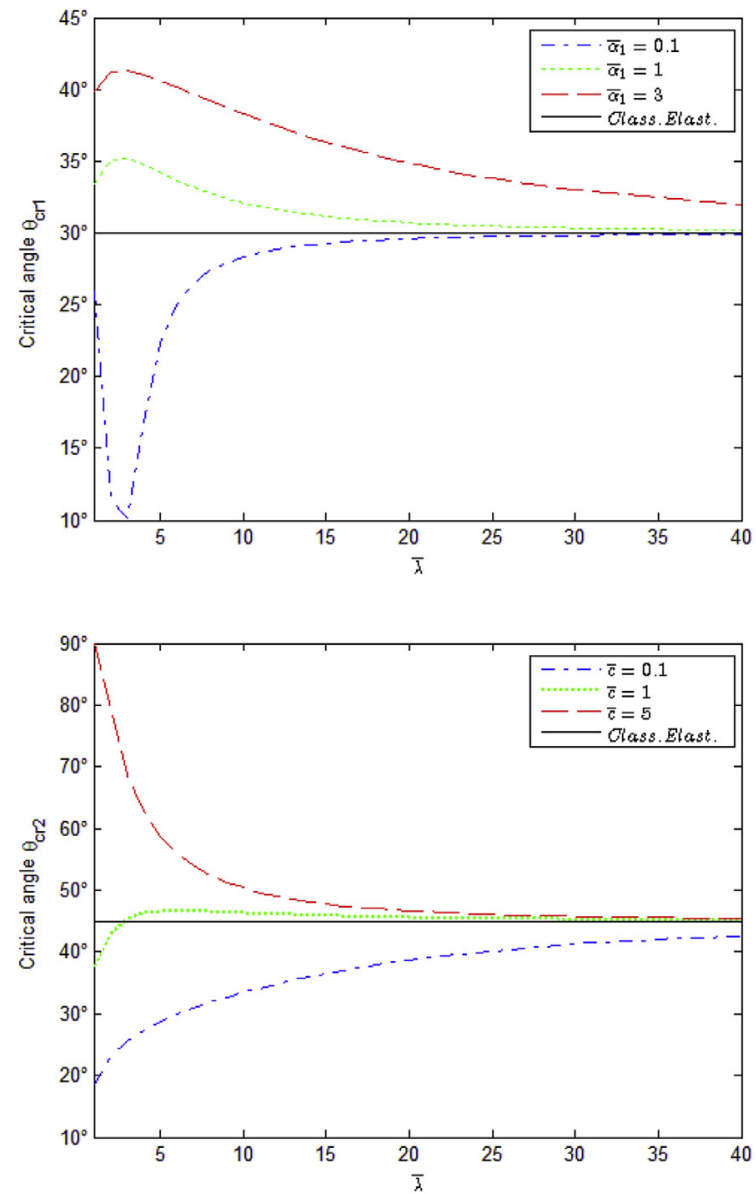

In this paper, the influence of the surface parameter $b_{i y}$ and their ratio $\bar{b}\left(=b_{2 y} / b_{1 y}\right)$ on the reflection and transmission waves are our main concerns.

Energy flux density of a plane wave along the propagation direction $\mathbf{n}$ can be obtained by,

$q(\mathbf{n}, t)=-P_{i}(\mathbf{n}) \dot{u}_{i}-R_{i}(\mathbf{n}) n_{j} \dot{u}_{i, j}$.

Due to the time dependence of energy flux, the average energy flux over one period, namely, $\bar{q}(\mathbf{n})=1 / T \int_{0}^{T} q(\mathbf{n}, t) d t$, is more interesting and can be calculated for various waves.
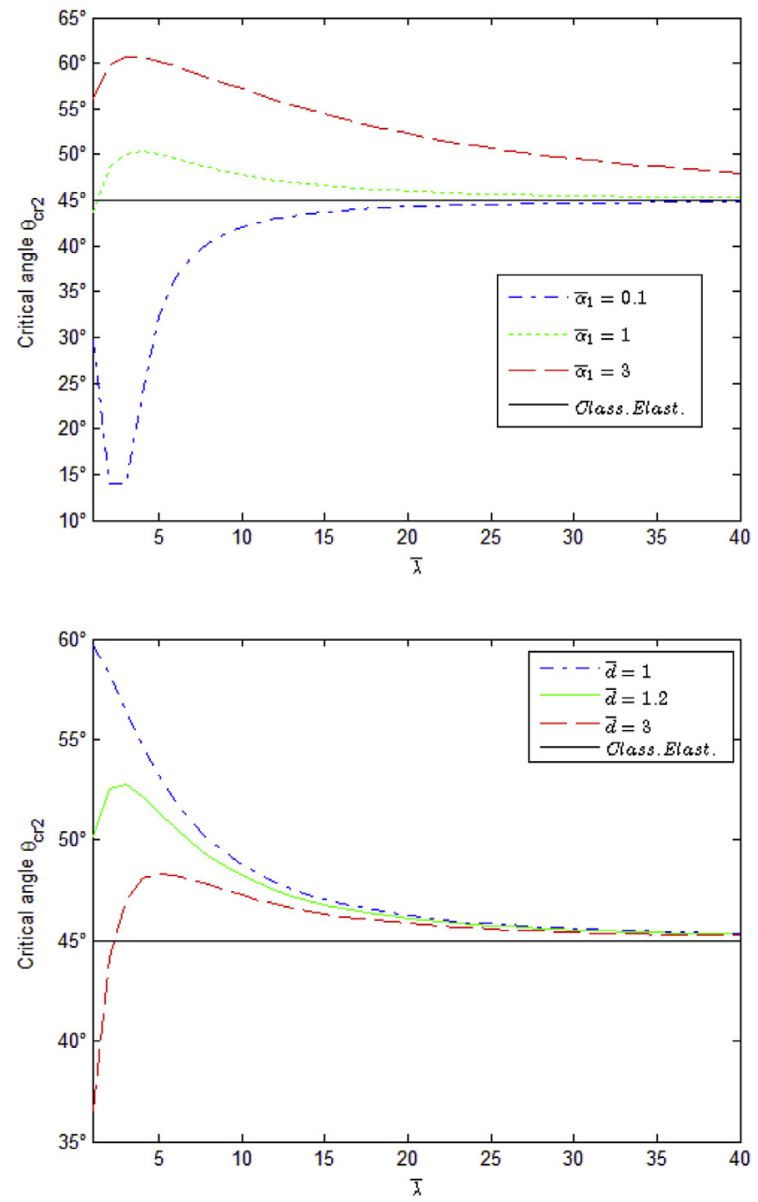

Fig. 9. Critical angles under an incident SV-wave for various microstructure parameter ratio $\alpha_{1}$ ( $b_{1}$ and $\bar{b}$ have no influence on the critical angle). 


$$
\begin{aligned}
\bar{q}_{0}^{p}\left(\mathbf{n}_{0}\right)= & \frac{1}{2} \omega \sigma_{p 1}^{3}\left[\left(\lambda_{1}+2 \mu_{1}\right)-\mu_{1} m_{1}\right. \\
& \left.+2 c_{1}\left(\lambda_{1}+2 \mu_{1}\right) \sigma_{p 1}^{2}\right] A_{1}^{(0)} A_{1}^{(0)^{*}} \\
\bar{q}_{0}^{s}\left(\mathbf{n}_{0}\right)= & \frac{1}{2} \omega \sigma_{s 1}^{3} \mu_{1}\left(1-m_{1}+2 c_{1} \sigma_{s 1}^{2}\right) B_{1}^{(0)} B_{1}^{(0)^{*}} \\
\bar{q}_{1}^{p 1}\left(\mathbf{n}_{P 1}\right)= & \frac{1}{2} \omega \sigma_{p 1}^{3}\left[\left(\lambda_{1}+2 \mu_{1}\right)-\mu_{1} m_{1}\right. \\
& \left.+2 c_{1}\left(\lambda_{1}+2 \mu_{1}\right) \sigma_{p 1}^{2}\right] A_{2}^{(1)} A_{2}^{(1)^{*}} \\
\bar{q}_{2}^{p 2}\left(\mathbf{n}_{P 2}\right)= & \frac{1}{2} \omega \sigma_{p 2}^{3}\left[\left(\lambda_{2}+2 \mu_{2}\right)-\mu_{2} m_{2}\right. \\
& \left.+2 c_{2}\left(\lambda_{2}+2 \mu_{2}\right) \sigma_{p 2}^{2}\right] A_{2}^{(2)} A_{2}^{(2)^{*}} \\
\bar{q}_{1}^{s 1}\left(\mathbf{n}_{s 1}\right)= & \frac{1}{2} \omega \sigma_{s 1}^{3} \mu_{1}\left(1-m_{1}+2 c_{1} \sigma_{s 1}^{2}\right) B_{2}^{(1)} B_{2}^{(1)^{*}} \\
\bar{q}_{2}^{s 2}\left(\mathbf{n}_{s 2}\right)= & \frac{1}{2} \omega \sigma_{s 2}^{3} \mu_{2}\left(1-m_{2}+2 c_{2} \sigma_{s 2}^{2}\right) B_{2}^{(2)} B_{2}^{(2)^{*}}
\end{aligned}
$$
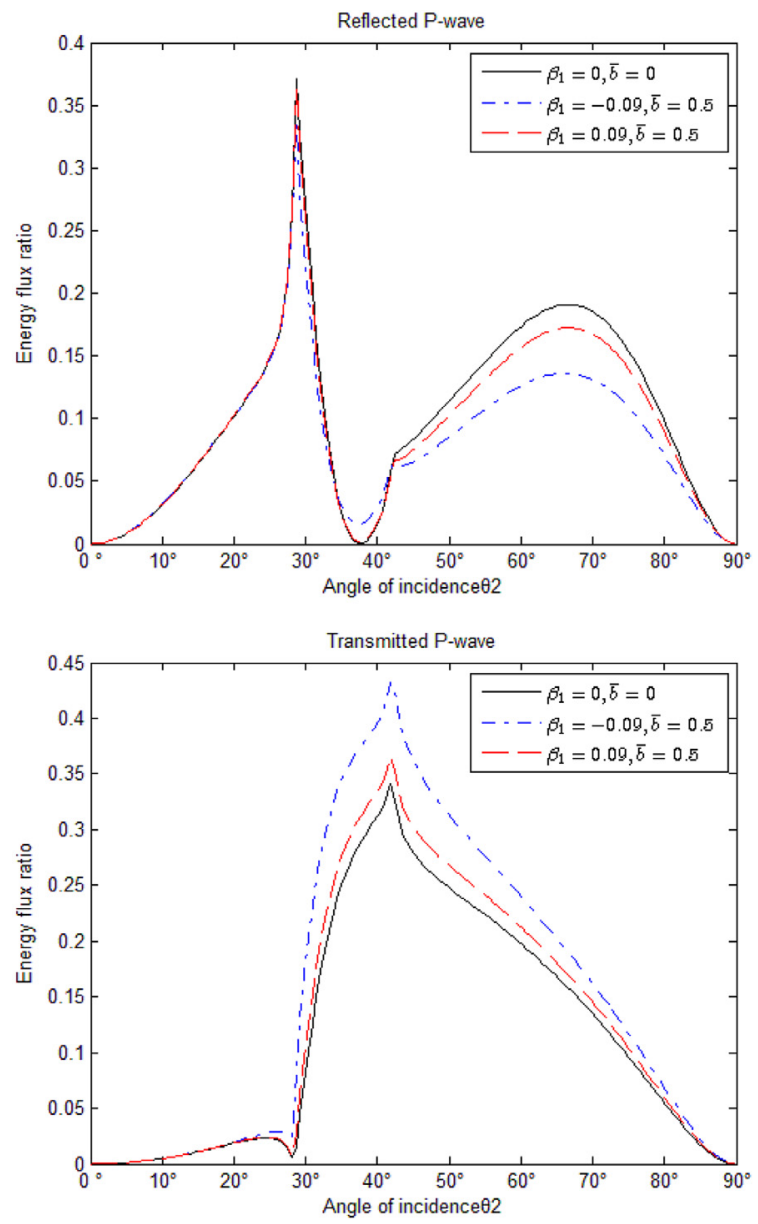

Fig. 10. The reflection and transmission coefficients of body waves under an incident SV wave for different $\beta_{1}\left(\alpha_{1}=0.1, \bar{\lambda}=5\right)$.
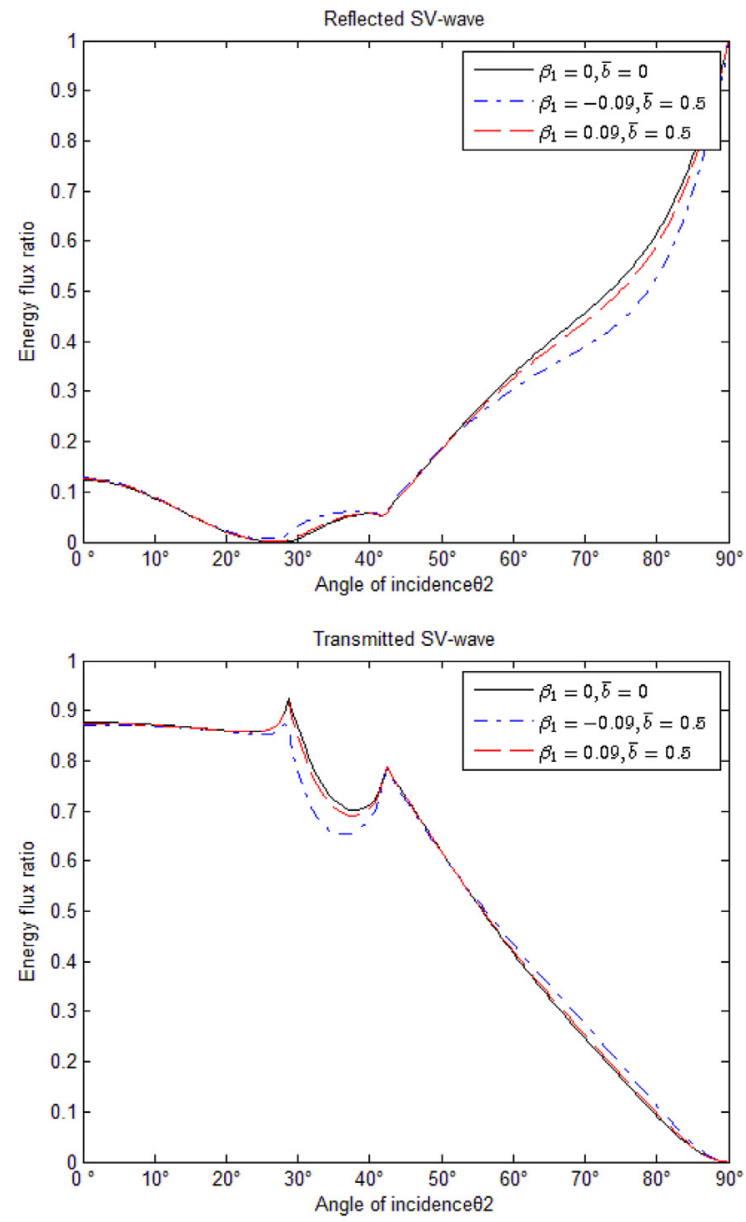

$$
\begin{aligned}
& m_{1}=a_{s 1}, \quad m_{2}=a_{s 2}, \quad \lambda_{i}=\frac{E_{i} \gamma_{i}}{\left(1+\gamma_{i}\right)\left(1-2 \gamma_{i}\right)}, \\
& \mu_{i}=\frac{E_{i}}{2\left(1+\gamma_{i}\right)}, \quad(i=1,2) .
\end{aligned}
$$

For the SP and SS surface waves, the energy flux density on the wavefront decreases gradually with the increase of $z$. Here, a unit area on the wavefront is taken to be $l_{z} \times l_{y}=\gamma_{p} \times 1 / \gamma_{p}$ or $l_{z} \times l_{y}=\gamma_{s} \times 1 / \gamma_{s}$ near the surface, which is elaborately chosen to make $\gamma_{p}$ or $\gamma_{s}$ disappear in the expression of energy flux. Then, the energy flux density of surface waves can be expressed as

$\bar{q}_{1}^{s p 1}(\mathbf{n})=\frac{1}{2} M \omega \xi_{1}\left(C_{1}^{(1)} C_{1}^{(1)}\right) J_{1}^{s p 1}$

$\bar{q}_{1}^{s S 1}(\mathbf{n})=\frac{1}{2} M \omega \zeta_{1}\left(C_{2}^{(1)} C_{2}^{(1) \cdot}\right) J_{1}^{s S 1}$

$\bar{q}_{2}^{s p 2}(\mathbf{n})=\frac{1}{2} M \omega \xi_{2}\left(C_{1}^{(2)} C_{1}^{(2) \cdot}\right) J_{2}^{s p 2}$

$\bar{q}_{2}^{s s 2}(\mathbf{n})=\frac{1}{2} M \omega \zeta_{2}\left(C_{2}^{(2)} C_{2}^{(2) \cdot}\right) J_{2}^{s s 2}$, 
where

$M=\frac{1-\exp (-2)}{2}$,

$$
\begin{aligned}
J_{1}^{s p 1}= & \lambda_{1} \tau_{p 1}^{2}-2 \mu_{1}\left(\tau_{p 1}^{2}+\xi_{1}^{2}\right)+\mu_{1} m_{1}\left(\tau_{p 1}^{2}+2 \xi_{1}^{2}\right)+2 c_{1} \tau_{p 1}^{2}\left[\lambda_{1} \xi_{1}^{2}\right. \\
& \left.+2 \mu_{1}\left(\tau_{p 1}^{2}+2 \xi_{1}^{2}\right)\right],
\end{aligned}
$$

$$
\begin{aligned}
J_{1}^{s s 1}= & \mu_{1}\left[-\left(3 \tau_{s 1}^{2}+4 \zeta_{1}^{2}\right)+m_{1}\left(\tau_{s 1}^{2}+2 \zeta_{1}^{2}\right)+2 c_{1} \tau_{s 1}^{2}\left(2 \tau_{s 1}^{2}\right.\right. \\
& \left.\left.+3 \zeta_{1}^{2}\right)\right]
\end{aligned}
$$

$$
\begin{aligned}
J_{2}^{s p 2}= & \lambda_{2} \tau_{p 2}^{2}-2 \mu_{2}\left(\tau_{p 2}^{2}+\xi_{2}^{2}\right)+\mu_{2} m_{2}\left(\tau_{p 2}^{2}+2 \xi_{2}^{2}\right)+2 c_{2} \tau_{p 2}^{2}\left[\lambda_{2} \xi_{2}^{2}\right. \\
& \left.+2 \mu_{2}\left(\tau_{p 2}^{2}+2 \xi_{2}^{2}\right)\right] \\
J_{2}^{s s 2}= & \mu_{2}\left[-\left(3 \tau_{s 2}^{2}+4 \zeta_{2}^{2}\right)+m_{2}\left(\tau_{s 2}^{2}+2 \zeta_{2}^{2}\right)+2 c_{2} \tau_{s 2}^{2}\left(2 \tau_{s 2}^{2}+3 \zeta_{2}^{2}\right)\right],
\end{aligned}
$$

Define the reflection and transmission coefficients as the energy flux ratio along the propagation directions of various reflection waves and transmission waves with respect to incident waves, namely, $\quad \bar{q}_{1}^{p 1}\left(\mathbf{n}_{p 1}\right) / \bar{q}_{0}^{p}\left(\mathbf{n}_{0}\right), \quad \bar{q}_{1}^{s 1}\left(\mathbf{n}_{s 1}\right) / \bar{q}_{0}^{p}\left(\mathbf{n}_{0}\right), \quad \bar{q}_{1}^{s p 1}(\mathbf{n}) / \bar{q}_{0}^{p}\left(\mathbf{n}_{0}\right) \quad$ and $\bar{q}_{1}^{s s 1}(\mathbf{n}) / \bar{q}_{0}^{p}\left(\mathbf{n}_{0}\right)$ as the reflection coefficients and $\bar{q}_{1}^{p 2}\left(\mathbf{n}_{p 1}\right) / \bar{q}_{0}^{p}\left(\mathbf{n}_{0}\right)$, $\bar{q}_{1}^{s 2}\left(\mathbf{n}_{s 1}\right) / \bar{q}_{0}^{p}\left(\mathbf{n}_{0}\right), \bar{q}_{1}^{s p 2}(\mathbf{n}) / \bar{q}_{0}^{p}\left(\mathbf{n}_{0}\right)$ and $\bar{q}_{1}^{s s 2}(\mathbf{n}) / \bar{q}_{0}^{p}\left(\mathbf{n}_{0}\right)$ as the transmission coefficients in the case of incident $P$ wave. In the case of incident SV wave, the denominator should be replaced by $\bar{q}_{0}^{s}\left(\mathbf{n}_{0}\right)$. Consider that the energy flux vectors of various surface waves are along the interface, the energy conservation law requires

$E=\frac{\bar{q}^{p 1}\left(\mathbf{n}_{P 1}\right) \cos \theta_{p 1}+\bar{q}^{s 1}\left(\mathbf{n}_{s 1}\right) \cos \theta_{s 1}+\bar{q}^{p 2}\left(\mathbf{n}_{p 2}\right) \cos \theta_{p 2}+\bar{q}^{s 2}\left(\mathbf{n}_{s 2}\right) \cos \theta_{s 2}}{\bar{q}_{0}\left(\mathbf{n}_{0}\right) \cos \theta}=1$,
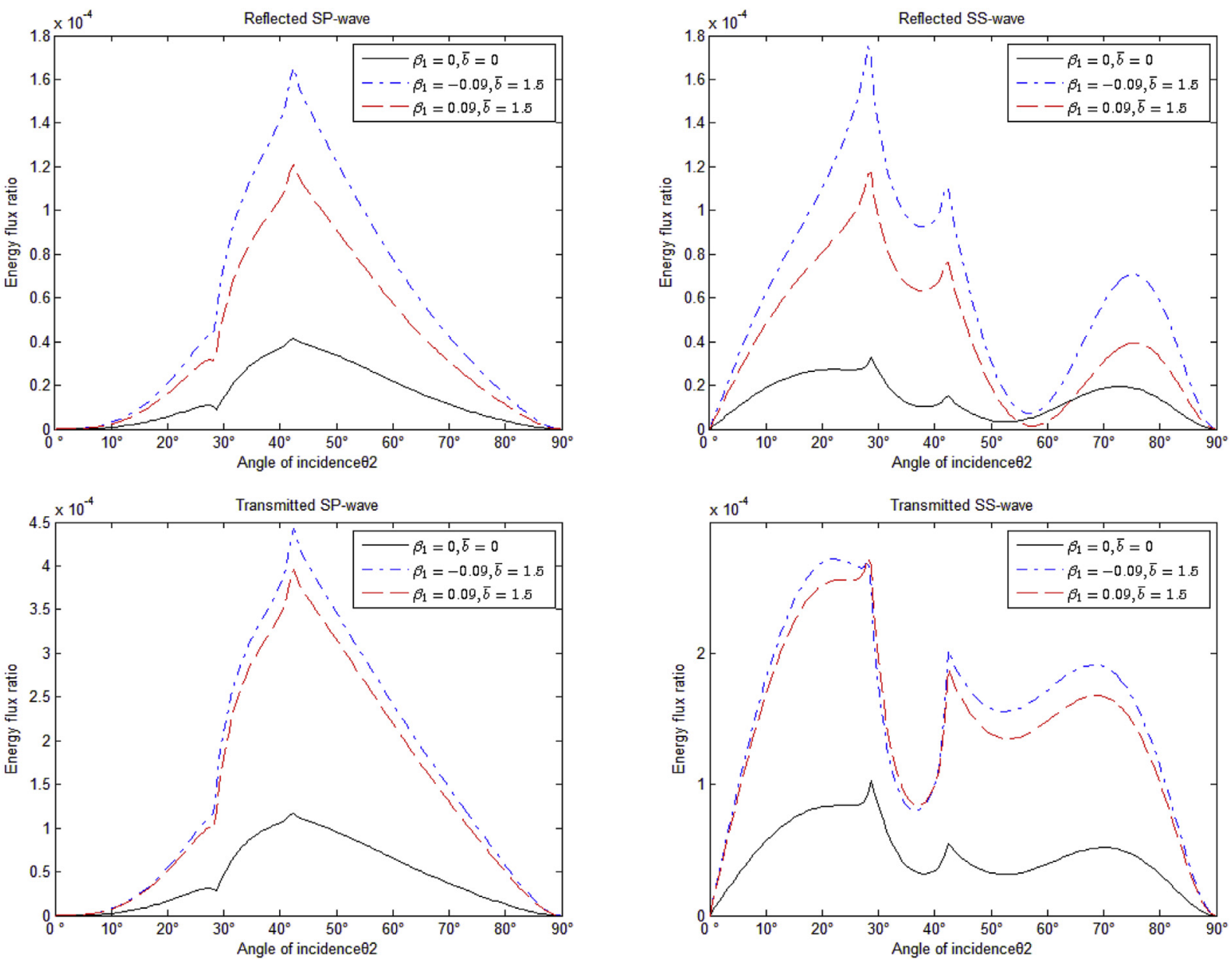

Fig. 11. The reflection and transmission coefficients of surface waves under an incident SV wave for different $\beta_{1}\left(\alpha_{1}=0.1, \bar{\lambda}=5\right)$. 
It means that the energy flux of incident wave (input energy flux) through a unit area at interface is equal to the energy flux of reflection and transmission waves (output energy flux) through same area at interface. Eq. (36) is used to validate the numerical results in the next section.

\section{Numerical examples and discussion}

In the numerical examples, the main concerns are focused on the influences of the surface parameter $b_{1 y}$ and $b_{2 y}$ of two microstructure solids on the reflection and transmission coefficients. The Poisson's ratio of medium 1 and medium 2 is taken to be $\nu_{1}=\nu_{2}=1$ / 3 . The density ratio of them is taken to be $\bar{\rho}\left(=\rho_{2} / \rho_{1}\right)=2 / 3$. The modulus ratio is taken to be $\bar{E}\left(=E_{2} / E_{1}\right)=1 / 3$. The microstructure parameter ratios are taken to be $\bar{c}=1.4$ and $\bar{d}=1.4$.

\subsection{Incident $P$ wave case}

Fig. 2 shows the reflection and transmission coefficients in terms of the energy flux ratio for different surface parameter $b_{1 y}$. It is observed that the SV wave is more sensitive to $b_{1 y}$ than the $\mathrm{P}$ wave although the surface energy of solid influences the reflection and transmission of both body waves. Furthermore, both reflection and transmission coefficients of SV wave increase when the surface effects are taken into consideration no matter positive or negative $b_{1 y}$. This means that the surface effects can increase the mode conversion. Fig. 3 shows the influence of surface parameter $b_{1 y}$ on the surface waves. Although the energy carried by the surface wave is smaller about three orders of magnitude than that carried by the body wave, the influences of surface energy on the surface waves are the most evident. The energy carried by any surface wave, no matter reflection or transmission waves, P-type surface wave or Stype surface wave, increases when the surface energy effects are taken into consideration.

Figs. 4 and 5 show the influences of the ratio of two surface parameters $\left(\bar{b}=b_{2 y} / b_{1 y}\right)$ on the reflection and transmission coefficients. It is observed that the reflected P wave and the transmitted SV wave increase whereas the reflected SV wave and the transmitted $\mathrm{P}$ wave decrease when the ratio of two surface parameter $\bar{b}$ increases. However, the reflection and transmission coefficients of any surface wave, no matter P-type or S-type surface waves, increase monotonously with the increase of $\bar{b}$, see Fig. 5 .

Figs. 6 and 7 show the dependence of the reflection and transmission coefficients on the incident wavelength. As it is expected, the reflection and transmission coefficients of body waves tend to that of classical elastic solids and the surface waves disappear when the incident wavelength increases gradually. Only when the incident wavelength is close to the characteristic length of microstructure, the microstructure effects and the surface effects become notable. Moreover, the SV body waves are more sensitive to the incident wavelength than $P$ body wave. And the reflection and transmission coefficients of SV body wave increase when the incident wavelength becomes shorter. However, the reflection coefficients of $P$ wave increases while the transmission coefficients of $P$ wave decreases when the incident wavelength becomes shorter.
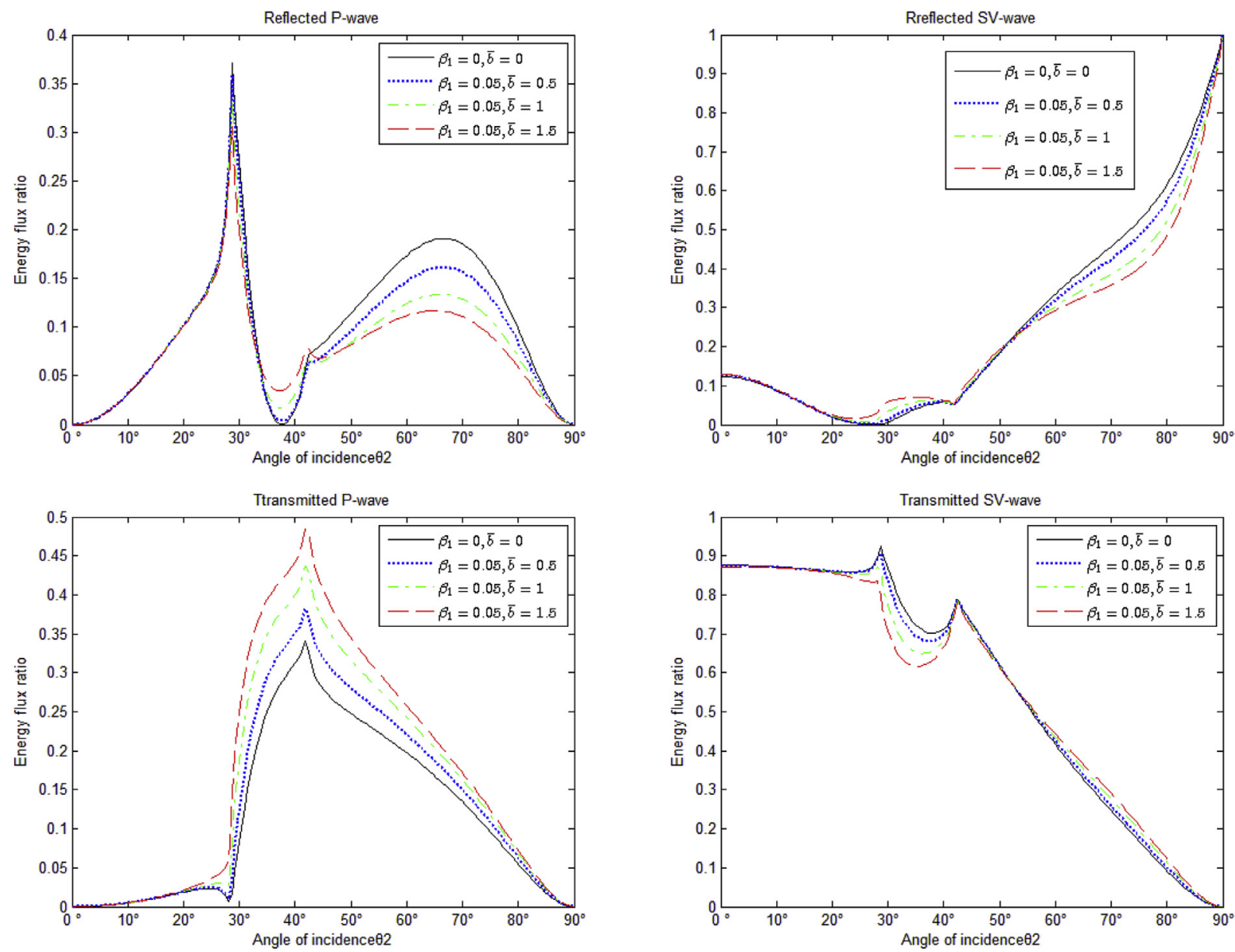

Fig. 12. The reflection and transmission coefficients of body waves under an incident SV-wave for different $\bar{b}\left(\beta_{1}=0.05, \alpha_{1}=0.1, \bar{\lambda}=5\right)$. 
Fig. 8 shows the reflection and transmission coefficients in the case of normal incident $P$ wave. Due to no wave mode conversion and the disappearance of the surface waves in the normal incidence. Only the reflection and transmission $P$ waves exist. There are two groups of interface conditions that can be used to determine the reflection and transmission coefficients as discussed in the previous Section. Fig. 8 shows the numerical results obtained from group B and the inset shows the numerical results obtained from group A. The interface conditions in group B include the surface effects on the reflection and transmission coefficients but the reflection and transmission coefficients do not tend to that of classical elastic solids when the incident wavelength increase gradually. On the contrary, the interface conditions in group A do not include the surface effects on the reflection and transmission coefficients but the reflection and transmission coefficients asymptotically tend to that of classical elastic solids when the incident wavelength increase gradually.

\subsection{Incident SV wave case}

In the case of incident SV wave, there are two critical angles for the given material constants combination. The first critical angles is corresponding with that the reflection body $\mathrm{P}$ wave becomes into surface wave and the second critical angle is corresponding with that the transmission body P wave becomes into surface wave. The microstructure parameter ratio has evident influence on the critical angles when the incident wavelength is comparable with the microstructure characteristic length. The first critical angle is only dependent upon the $\alpha_{1}$ while the second critical angle is also
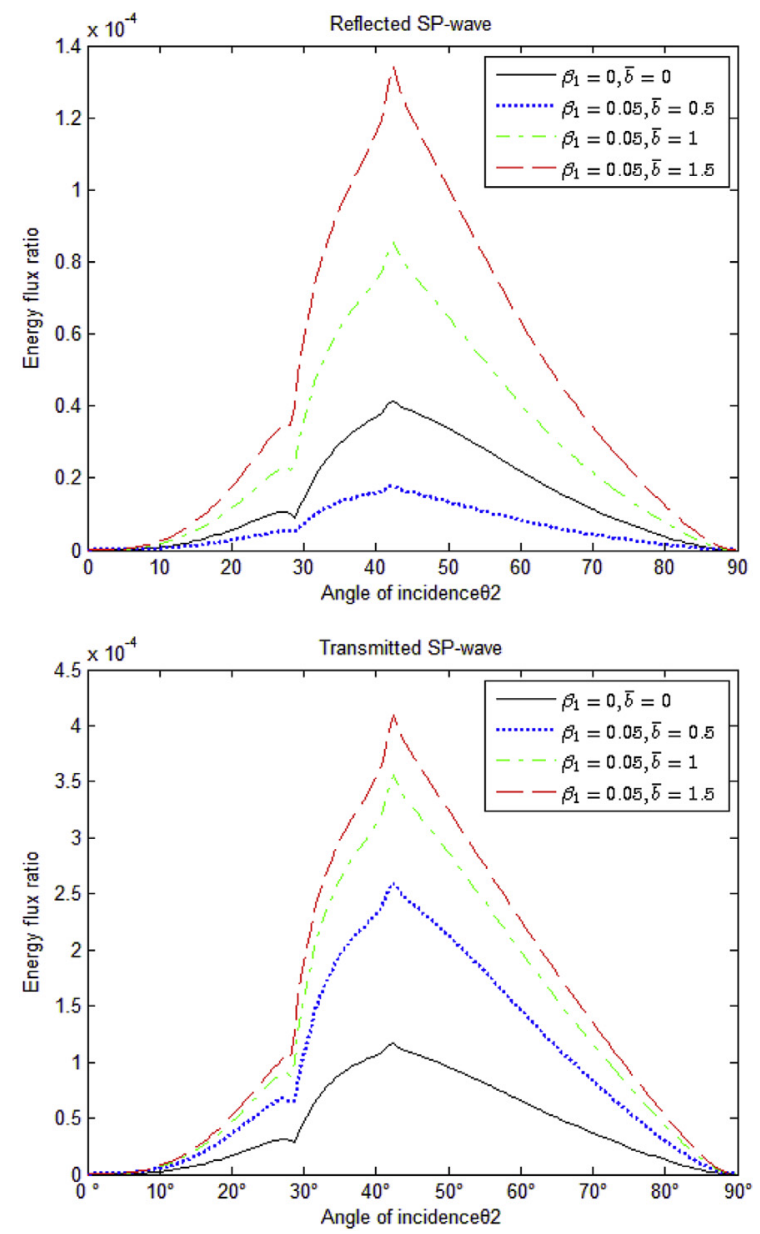

dependent upon $\bar{c}$ and $\bar{d}$, see Fig. 9. However, both critical angles are not dependent of the $b_{1 y}$ and $\bar{b}$. This means that the surface effects do not influence the critical angle. It is also noted that the critical angles of gradient elastic solid may greater or smaller than that of classical elastic solid dependent upon the ratio of microstructure parameters. The existence of critical angles usually makes the amplitudes of reflection and transmission waves changing abruptly when the incident wave gets across the critical angle.

The reflection and transmission coefficients of body wave are shown in Fig. 10 and that of surface waves are shown in Fig. 11. The abrupt change of amplitude of reflection and transmission waves at two critical angles are observed as expected. It is noted that the surface waves are more sensitive to the surface parameter $b_{1 y}$ than the body waves. The energy carried by the surface waves, no matter SP wave or SS wave, reflection waves or transmission waves, increase evidently when the surface effects are taken into consideration. The $\mathrm{P}$ waves are more sensitive to the surface parameter $b_{1 y}$ compared with the SV waves. The reflection $\mathrm{P}$ wave decreases whereas transmission P wave increases due to the surface effects.

Figs. 12 and 13 show the influence of the ratio of surface parameter $\bar{b}$ on the body waves and the surface waves, respectively. Similar to the observation in incident $\mathrm{P}$ wave, the influence of the surface parameter ratio $\bar{b}$ is evidently larger on the surface waves than on the body waves. The reflection and transmission body waves may increase or decrease dependent up on the incident angle. However, the surface waves, no matter SP wave or SS wave, reflection or transmission wave, increase monotonously with the increase of the surface parameter ratio $\bar{b}$.
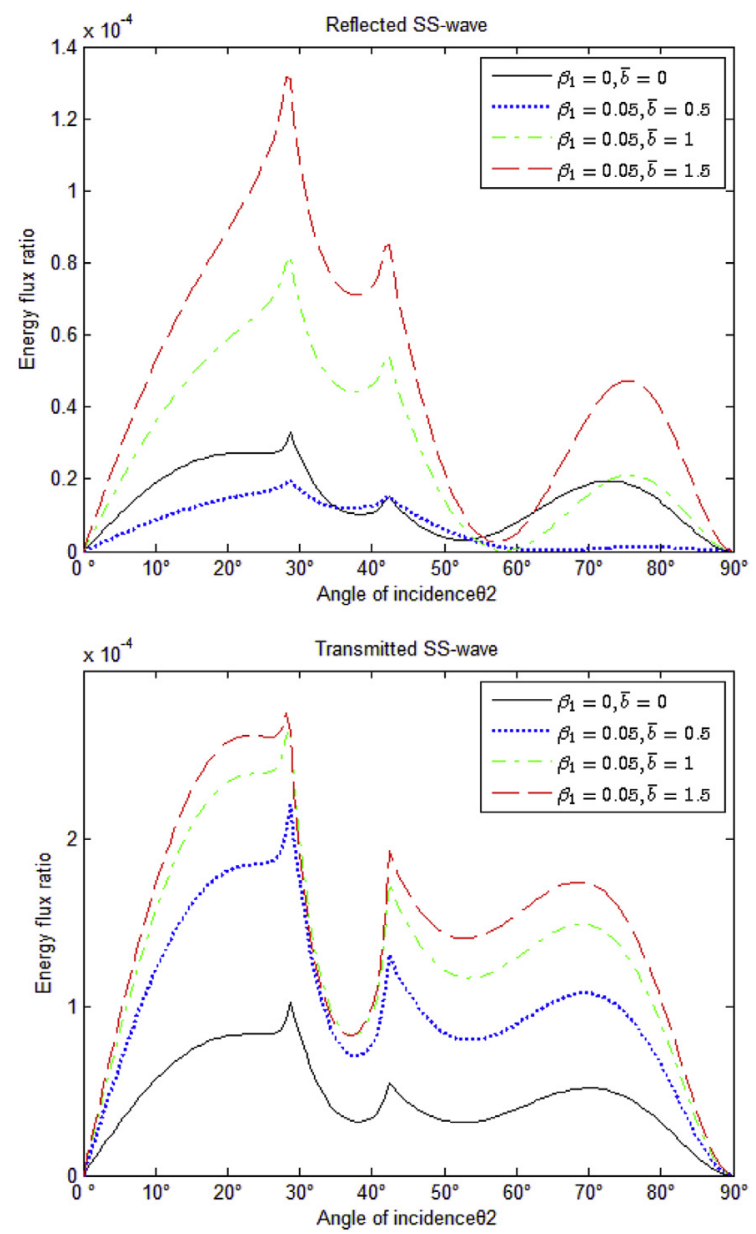

Fig. 13. The reflection and transmission coefficients of surface waves under an incident SV-wave for different $\bar{b}\left(\alpha_{1}=0.1, \bar{\lambda}=5\right)$. 

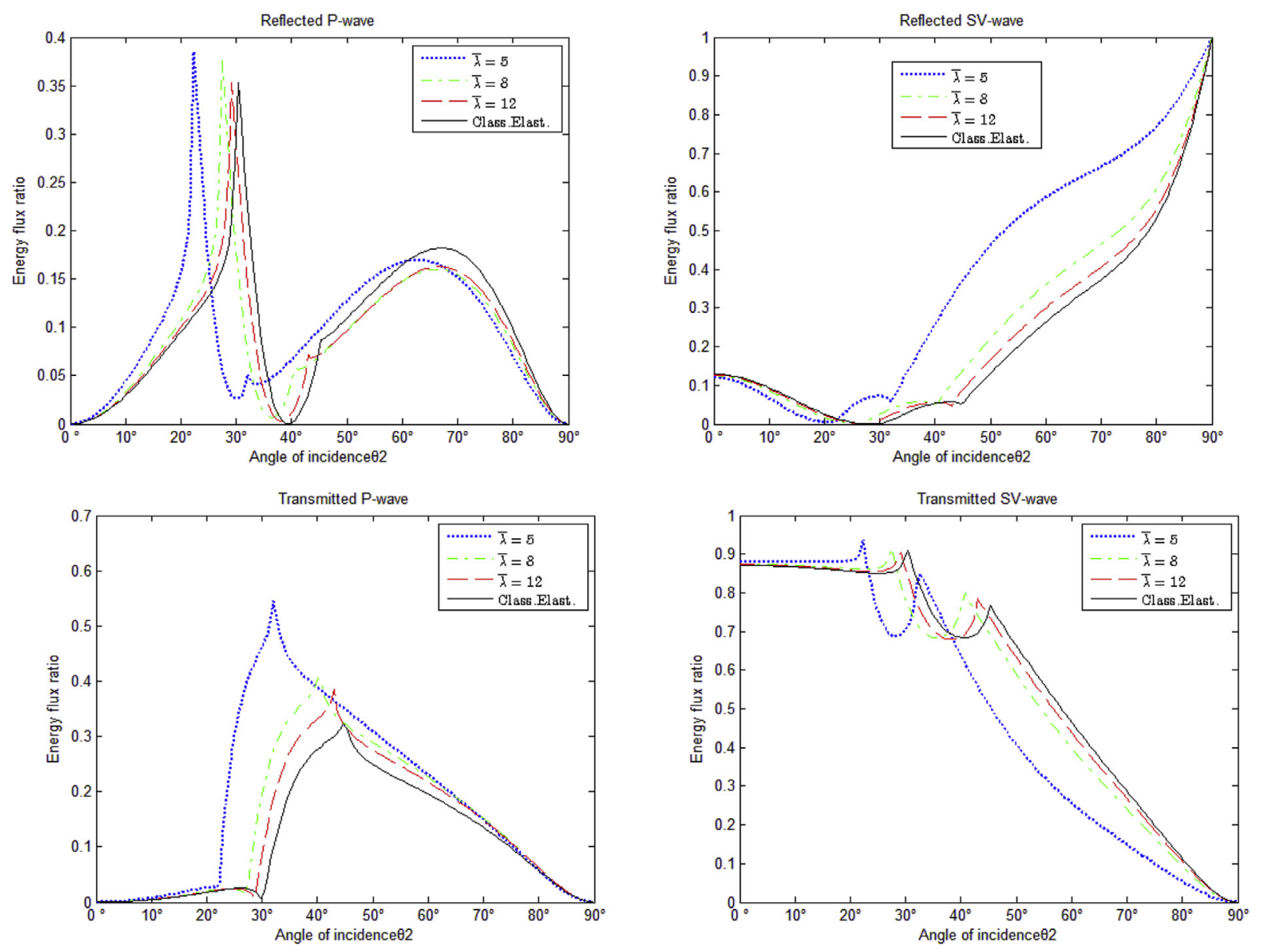

Fig. 14. The reflection and transmission coefficients of body waves under an incident SV-wave for different $\bar{\lambda}\left(\alpha_{1}=0.1, \bar{b}=1, \beta_{1}=-0.05\right)$.

Figs.14 and 15 show the influence of incident wavelength on the body waves and surface waves. Similar to the observation in the case of incident $P$ wave, the reflection and transmission body waves reduce to that in the classical elastic solids when the incident wavelength increase gradually. The surface waves, no matter reflection wave or transmission wave, decrease monotonously till to disappearance when the incident wavelength increase gradually.

In the case of normal incident SV wave, only the reflection and transmission SV waves exist due to no wave mode conversion and the disappearance of the surface waves. As discussed in normal incidence P wave, there are two groups of interface conditions that can be used to determine the reflection and transmission coefficients. The reflection and transmission coefficients obtained from the interface conditions in group B can reflect the surface effects but do not tend to that of classical elastic solids when the wavelength increase gradually. The reflection and transmission coefficients obtained from the interface conditions in group A cannot reflect the surface effects but can asymptotically tend to that of classical elastic solids when the wavelength increase gradually. The numerical results obtained for a normal incident SV wave are very similar to that for a normal incident $\mathrm{P}$ wave and thus are not given here anymore.

\subsection{Verification of energy conservation}

In order to validate the numerical results, the energy conservation between the incident waves, reflection waves and the transmission waves are examined. The energy conservation requires the total energy flowing out from a unit area at interface equal to the total energy flowing in the same area at interface. In other word, the total energy taken away by the reflection and transmission waves is equal to the total energy taken in by the incident waves. Because the energy flux of surface waves is along the interfaces, only body waves are examined. Fig. 16 shows the energy conservation factor $E(E=1$ stands for the perfect conservation) in the case of incident $\mathrm{P}$ wave. It is observed that $E$ is very close to 1 with error within $0.1 \%$ for various cases in the present numerical examples. Fig. 17 shows the energy conservation factor in the case of incident SV wave. Although the energy conservation has a relative large deviation around two critical angles, the largest deviation is still within $0.2 \%$ for various cases in the present numerical examples.

\section{Conclusions}

The microstructure effects of the wave motion needs to be considered when the wavelength of incident wave is comparable to the characteristic length of microstructure. For the micro and nano scale devices, the surface effects apart from the microstructure effects are also not neglectable. In order to consider the surface effects, a thin membrane adhere to the bulk material without slipping but with different elastic properties from the bulk material, or even, a separate surface with its own bending stiffness, mass and inertia, are usually assumed. In this paper, the surface effects are incorporated into the constitutive relation of bulk material by the direct postulation of a specific function of strain energy density. 



Fig. 15. The reflection and transmission coefficients of surface waves under an incident SV-wave for different $\bar{\lambda}\left(\alpha_{1}=0.1, \bar{b}=1, \beta_{1}=-0.05\right)$.

Based on this method, the influences of surface effects on the reflection and transmission waves at interface of biomaterial with microstructures are calculated. Some conclusions can be drawn from the numerical results.

1) There are two body waves (P wave and SV wave) and two surface waves (SP wave and SS wave) for the reflection waves and transmission waves, respectively. The appearance of the surface waves is due to the microstructure effects. The introduction of surface energy does not change the wave propagation mode in the interior of solid and at the interface but change the amplitude of them.

2) The influences of surface energy on the surface waves are more evident than on the body waves. The energy carried by any surface wave, no matter reflection or transmission waves, SP wave or SS wave, increases obviously when the surface energy effects are taken into consideration.
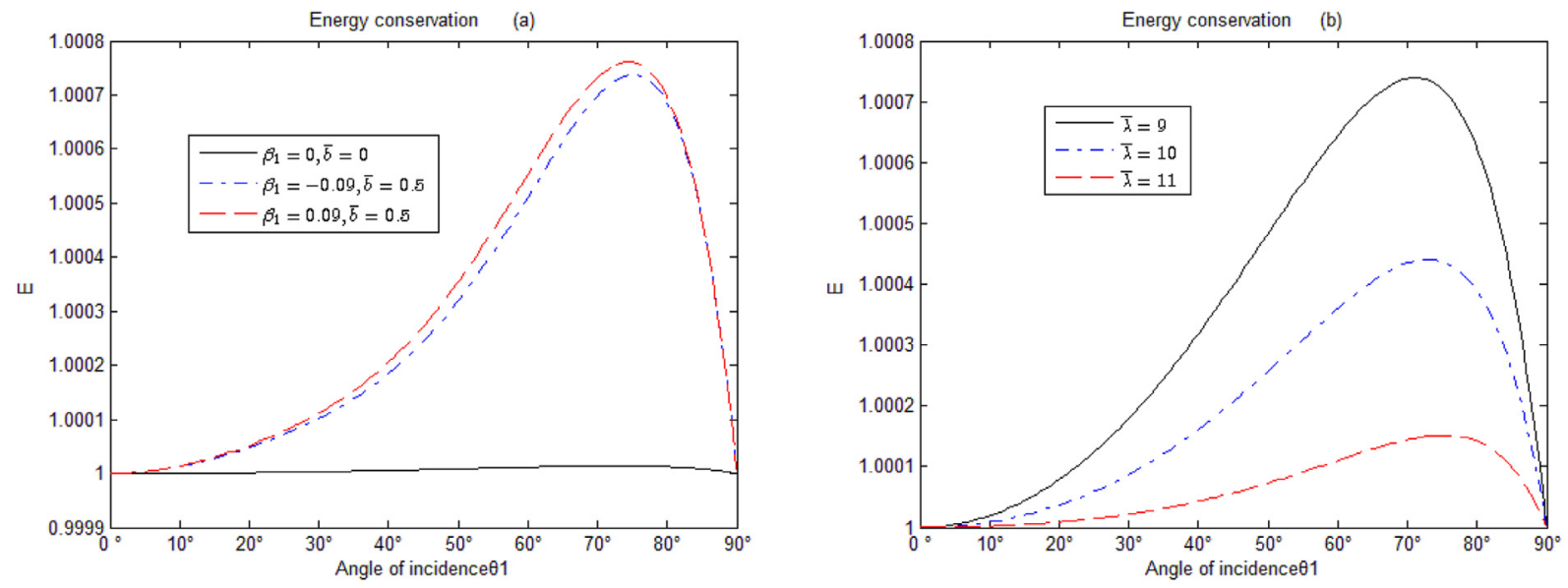

Fig. 16. Verification of energy conservation in the case of incident $\mathrm{P}$ wave. a) For different $\beta_{1}$; b) for different $\bar{\lambda}$. 

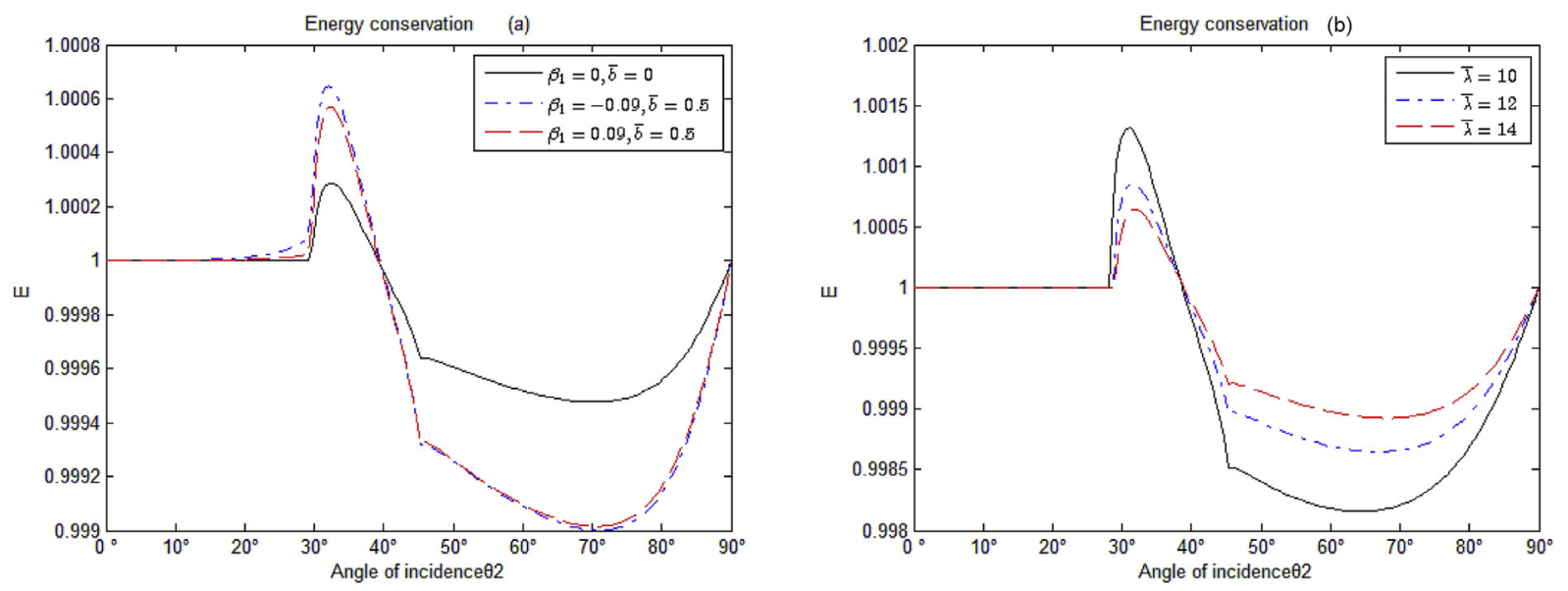

Fig. 17. Verification of energy conservation in the case of incident SV wave. a) For different $\beta_{1}$; b) for different $\bar{\lambda}$.

3) In the case of incident $P$ wave, reflection and transmission SV wave are more sensitive to the surface effects than P wave; In the case of incident SV wave, reflection and transmission $\mathrm{P}$ waves are more sensitive to the surface effects than SV waves. In particular, in the case of incident $P$ wave, the amplitude of both reflection and transmission SV waves increase when the surface effects are considered. This means that the surface effects help to the mode conversion.

4) The microstructure effects influence the critical angles and can make the critical angle greater or smaller than that of classical elastic solids, but the surface effects do not change the critical angles.

5) The microstructure effects and the surface effects are evident only when the wavelength of incident wave is comparable to the characteristic length of microstructure. With the increase of incident wavelength, the surface effects and the microstructure effects decrease gradually. The surface waves tend to disappearance and the body waves tend to that of classical elastic solids.

6) In the case of normal incidence, no mode conversion exists and the surface waves disappear also. There are two groups of interface conditions can be used to determine the reflection and transmission coefficients of body waves. The two groups of interface conditions are corresponding with different surface microstructure. One of two groups does not include the surface effects and the other group includes the surface effects.

\section{Acknowledgement}

The work is supported by the National Natural Science Foundation of China (No.10972029) and Opening fund of State Key Laboratory of Nonlinear Mechanics (LNM)

\section{Appendix A}

The explicit expression of matrix $\mathbf{A}=\left(a_{i j}\right)_{8 \times 8}, \mathbf{B}=\left(b_{i j}\right)_{8 \times 1}$ and $\mathbf{C}=\left(c_{i j}\right)_{8 \times 1}$ in Eq. (30) are

$$
\begin{aligned}
& a_{31}=-\xi_{1} \beta_{p 1}, \quad a_{32}=-\xi_{1} \gamma_{p 1} i, \quad a_{33}=-\beta_{s 1}^{2}, \quad a_{34}=\gamma_{s 1}^{2}, \\
& a_{35}=-\xi_{2} \beta_{p 2}, \quad a_{36}=-\xi_{2} \gamma_{p 2} i, \quad a_{37}=\beta_{s 2}^{2}, \quad a_{38}=-\gamma_{s 2}^{2}, \\
& a_{41}=-\beta_{p 1}^{2}, \quad a_{42}=\gamma_{p 1}^{2}, \quad a_{43}=\zeta_{1} \beta_{s 1}, \quad a_{44}=\zeta_{1} \gamma_{s 1} i, \\
& a_{45}=\beta_{p 2}^{2}, \quad a_{46}=-\gamma_{p 2}^{2}, \quad a_{47}=\zeta_{2} \beta_{s 2}, \quad a_{48}=\zeta_{2} \gamma_{s 2} i, \\
& a_{51}=\mu_{1}\left[-2 \xi_{1} \beta_{p 1}+2 b_{y}^{(1)} i \xi_{1}\left(\xi_{1}^{2}+\sigma_{p 1}^{2}\right)-2 c_{1} \xi_{1} \beta_{p 1}\left(2 \sigma_{p 1}^{2}+\xi_{1}^{2}\right)\right. \\
& \left.+\frac{d_{1}^{2} \omega^{2}}{3 V_{s 1}^{2}} \xi_{1} \beta_{p 1}\right],
\end{aligned}
$$

$$
\begin{aligned}
a_{52}= & \mu_{1}\left[-2 \xi_{1} \gamma_{p 1} i-2 b_{y}^{(1)} i \xi_{1}\left(\tau_{p 1}^{2}-\xi_{1}^{2}\right)+2 c_{1} \xi_{1} \gamma_{p 1}\left(2 \tau_{p 1}^{2}\right.\right. \\
& \left.\left.-\xi_{1}^{2}\right) i+\frac{d_{1}^{2} \omega^{2}}{3 V_{s 1}^{2}} \xi_{1} \gamma_{p 1} i\right]
\end{aligned}
$$$$
a_{53}=\mu_{1}\left[\left(\zeta_{1}^{2}-\beta_{s 1}^{2}+2 b_{y}^{(1)} \zeta_{1}^{2} \beta_{s 1} i\right)-c_{1}\left(\beta_{s 1}^{4}-\zeta_{1}^{4}+2 \zeta_{1}^{2} \beta_{s 1}^{2}\right)\right.
$$$$
\left.+\frac{d_{1}^{2} \omega^{2}}{3 V_{s 1}^{2}} \beta_{s 1}^{2}\right]
$$$$
a_{54}=\mu_{1}\left[\left(\zeta_{1}^{2}+\gamma_{s 1}^{2}-2 b_{y}^{(1)} \zeta_{1}^{2} \gamma_{s 1}\right)-c_{1}\left(\gamma_{s 1}^{4}-\zeta_{1}^{4}-2 \zeta_{1}^{2} \gamma_{s 1}^{2}\right)\right.
$$$$
\left.-\frac{d_{1}^{2} \omega^{2}}{3 V_{s 1}^{2}} \gamma_{s 1}^{2}\right]
$$

$a_{11}=\xi_{1}, \quad a_{12}=\xi_{1}, \quad a_{13}=\beta_{s 1}, \quad a_{14}=\gamma_{s 1} i, \quad a_{15}=-\xi_{2}, \quad a_{16}=-\xi_{2}, \quad a_{17}=\beta_{s 2}, \quad a_{18}=\gamma_{s 2} i$ $a_{21}=\beta_{p 1}, \quad a_{22}=\gamma_{p 1} i, \quad a_{23}=-\zeta_{1}, \quad a_{24}=-\zeta_{1}, \quad a_{25}=\beta_{p 2}, \quad a_{26}=\gamma_{p 2} i, \quad a_{27}=\zeta_{2}, \quad a_{28}=\zeta_{2}$, 


$$
\begin{aligned}
& a_{55}=-\mu_{2}\left[2 \xi_{2} \beta_{p 2}+2 b_{y}^{(2)} i \xi_{2}\left(\xi_{2}^{2}+\sigma_{p 2}^{2}\right)+2 c_{2} \xi_{2} \beta_{p 2}\left(2 \sigma_{p 2}^{2}+\xi_{2}^{2}\right) \quad a_{66}=-\mu_{2}\left[2\left(2 \tau_{p 2}^{2}+\xi_{p 2}^{2}\right)+2 b_{y}^{(2)} \xi_{2}^{2} \gamma_{p 2}\right.\right. \\
& \left.-\frac{d_{2}^{2} \omega^{2}}{3 V_{s 2}^{2}} \xi_{2} \beta_{p 2}\right] \\
& \left.-2 c_{2}\left(\xi_{2}^{4}-4 \xi_{2}^{2} \gamma_{p 2}^{2}+2 \gamma_{p 2}^{4}\right)-\frac{d_{2}^{2} \omega^{2}}{3 V_{s 2}^{2}} \gamma_{p 2}^{2}\right] \\
& a_{56}=-\mu_{2}\left[2 \xi_{2} \gamma_{p 2} i-2 b_{y}^{(2)} i \xi_{2}\left(\tau_{p 2}^{2}-\xi_{2}^{2}\right)-2 c_{2} \xi_{2} \gamma_{p 2}\left(2 \tau_{p 2}^{2}\right.\right. \\
& \left.\left.--\xi_{2}^{2}\right) i+\frac{d_{2}^{2} \omega^{2}}{3 V_{s 2}^{2}} \xi_{2} \gamma_{p 2} i\right] \\
& a_{57}=-\mu_{2}\left[\left(\zeta_{2}^{2}-\beta_{s 2}^{2}-2 b_{y}^{(2)} \zeta_{2}^{2} \beta_{s 2} i\right)-c_{2}\left(\beta_{s 2}^{4}-\zeta_{2}^{4}+2 \zeta_{2}^{2} \beta_{s 2}^{2}\right)\right. \\
& \left.+\frac{d_{2}^{2} \omega^{2}}{3 V_{s 2}^{2}} \beta_{s 2}^{2}\right] \\
& a_{58}=-\mu_{2}\left[\left(\zeta_{2}^{2}+\gamma_{s 2}^{2}+2 b_{y}^{(2)} \zeta_{2}^{2} \gamma_{s 2}\right)-c_{2}\left(\gamma_{s 2}^{4}-\zeta_{2}^{4}-2 \zeta_{2}^{2} \gamma_{s 2}^{2}\right)\right. \\
& \left.-\frac{d_{2}^{2} \omega^{2}}{3 V_{s 2}^{2}} \gamma_{s 1}^{2}\right] \\
& a_{61}=\mu_{1}\left[-2\left(\sigma_{p 1}^{2}+\beta_{p 1}^{2}\right)+2 b_{y}^{(1)} \xi_{1}^{2} \beta_{p 1} i\right. \\
& \left.-2 c_{1}\left(\xi_{1}^{4}+4 \xi_{1}^{2} \beta_{p 1}^{2}+2 \beta_{p 1}^{4}\right)+\frac{d_{1}^{2} \omega^{2}}{3 V_{s 1}^{2}} \beta_{p 1}^{2}\right] \\
& a_{67}=-\mu_{2}\left[-2 \zeta_{2} \beta_{s 2}-b_{y}^{(2)} \zeta_{2}\left(\zeta_{2}^{2}-\beta_{s 2}^{2}\right) i-c_{2} \zeta_{2} \beta_{s 2}\left(\sigma_{s 2}^{2}+2 \zeta_{2}^{2}\right)\right. \\
& \left.+\frac{d_{2}^{2} \omega^{2}}{3 V_{s 2}^{2}} \zeta_{2} \beta_{s 2}\right] \\
& a_{68}=-\mu_{2}\left[2 \zeta_{2} \gamma_{s 2} i-b_{y}^{(2)} \zeta_{2}\left(\zeta_{2}^{2}+\gamma_{s 2}^{2}\right) i+c_{2} \zeta_{2} \gamma_{s 2}\left(\tau_{s 2}^{2}-2 \zeta_{2}^{2}\right) i\right. \\
& \left.+\frac{d_{2}^{2} \omega^{2}}{3 V_{s 2}^{2}} \zeta_{2} \gamma_{s 2} i\right] \\
& a_{71}=-2 \xi_{1} \beta_{p 1}\left(b_{y}^{(1)}+c_{1} \beta_{p 1} i\right) \mu_{1}, \\
& a_{72}=-2 \xi_{1} \gamma_{p 1}\left(b_{y}^{(1)}-c_{1} \gamma_{p 1}\right) i \mu_{1}, \\
& a_{73}=\left(\zeta_{1}^{2}-\beta_{s 1}^{2}\right)\left(b_{y}^{(1)}+c_{1} \beta_{s 1} i\right) \mu_{1}, \\
& a_{74}=\left(\zeta_{1}^{2}+\gamma_{s 1}^{2}\right)\left(b_{y}^{(1)}-c_{1} \gamma_{s 1}\right) \mu_{1}, \\
& a_{75}=-2 \xi_{2} \beta_{p 2}\left(b_{y}^{(2)}-c_{2} \beta_{p 2} i\right) \mu_{2}, \\
& a_{76}=-2 \xi_{2} \gamma_{p 2}\left(b_{y}^{(2)}+c_{2} \gamma_{p 2}\right) i \mu_{2}, \\
& a_{62}=\mu_{1}\left[2\left(2 \tau_{p 1}^{2}+\xi_{p 1}^{2}\right)-2 b_{y}^{(1)} \xi_{1}^{2} \gamma_{p 1}-2 c_{1}\left(\xi_{1}^{4}-4 \xi_{1}^{2} \gamma_{p 1}^{2}+2 \gamma_{p 1}^{4}\right)\right. \\
& \left.-\frac{d_{1}^{2} \omega^{2}}{3 V_{s 1}^{2}} \gamma_{p 1}^{2}\right] \\
& a_{77}=-\left(\zeta_{2}^{2}-\beta_{s 2}^{2}\right)\left(b_{y}^{(2)}-c_{2} \beta_{s 2} i\right) \mu_{2}, \\
& a_{78}=-\left(\zeta_{2}^{2}+\gamma_{s 2}^{2}\right)\left(b_{y}^{(2)}+c_{2} \gamma_{s 2}\right) \mu_{2} \text {, } \\
& a_{81}=-2 \mu_{1}\left(\sigma_{p 1}^{2}+\beta_{p 1}^{2}\right)\left(b_{y}^{(1)}+c_{1} \beta_{p 1} i\right), \\
& a_{82}=2 \mu_{1}\left(2 \tau_{p 1}^{2}+\xi_{p 1}^{2}\right)\left(b_{y}^{(1)}-c_{1} \gamma_{p 1}\right) \text {, } \\
& a_{63}=\mu_{1}\left[2 \zeta_{1} \beta_{s 1}-b_{y}^{(1)} \zeta_{1}\left(\zeta_{1}^{2}-\beta_{s 1}^{2}\right) i+c_{1} \zeta_{1} \beta_{s 1}\left(\sigma_{s 1}^{2}+2 \zeta_{1}^{2}\right)\right. \\
& a_{83}=2 \mu_{1} \zeta_{1} \beta_{s 1}\left(b_{y}^{(1)}+c_{1} \beta_{s 1} i\right), \\
& a_{84}=2 \mu_{1} \zeta_{1} \gamma_{s 1}\left(b_{y}^{(1)}-c_{1} \gamma_{s 1}\right) i \\
& a_{64}=\mu_{1}\left[2 \zeta_{1} \gamma_{s 1} i-b_{y}^{(1)} \zeta_{1}\left(\zeta_{1}^{2}+\gamma_{s 1}^{2}\right) i-c_{1} \zeta_{1} \gamma_{s 1}\left(\tau_{s 1}^{2}-2 \zeta_{1}^{2}\right) i\right. \\
& \left.-\frac{d_{1}^{2} \omega^{2}}{3 V_{s 1}^{2}} \zeta_{1} \gamma_{s 1} i\right] \\
& a_{85}=2 \mu_{2}\left(\sigma_{p 2}^{2}+\beta_{p 2}^{2}\right)\left(b_{y}^{(2)}-c_{2} \beta_{p 2} i\right) \\
& a_{86}=-2 \mu_{2}\left(2 \tau_{p 2}^{2}+\xi_{p 2}^{2}\right)\left(b_{y}^{(2)}+c_{2} \gamma_{p 2}\right) \text {, } \\
& a_{65}=-\mu_{2}\left[-2\left(\sigma_{p 2}^{2}+\beta_{p 2}^{2}\right)-2 b_{y}^{(2)} \xi_{2}^{2} \beta_{p 2} i\right. \\
& \left.-2 c_{2}\left(\xi_{2}^{4}+4 \xi_{2}^{2} \beta_{p 2}^{2}+2 \beta_{p 2}^{4}\right)+\frac{d_{2}^{2} \omega^{2}}{3 V_{s 2}^{2}} \beta_{p 2}^{2}\right] \\
& a_{87}=2 \mu_{2} \zeta_{2} \beta_{s 2}\left(b_{y}^{(1)}-c_{2} \beta_{s 2} i\right), \\
& a_{88}=2 \mu_{2} \zeta_{2} \gamma_{s 2}\left(b_{y}^{(2)}+c_{2} \gamma_{s 2}\right) i \\
& b_{11}=-\xi_{1}, \quad b_{21}=\beta_{p 1}, \quad b_{31}=-\xi_{1} \beta_{p 1}, \quad b_{41}=\beta_{p 1}^{2},
\end{aligned}
$$




$$
\begin{aligned}
& b_{51}=-\mu_{1}\left[2 \xi_{1} \beta_{p 1}+2 b_{y}^{(1)} i \xi_{1}\left(\xi_{1}^{2}+\sigma_{p 1}^{2}\right)+2 c_{1} \xi_{1} \beta_{p 1}\left(2 \sigma_{p 1}^{2}+\xi_{1}^{2}\right)\right. \\
& \left.-\frac{d_{1}^{2} \omega^{2}}{3 V_{s 1}^{2}} \xi_{1} \beta_{p 1}\right] \\
& b_{61}=\mu_{1}\left[2\left(\sigma_{p 1}^{2}+\beta_{p 1}^{2}\right)+2 b_{y}^{(1)} \xi_{1}^{2} \beta_{p 1} i+2 c_{1}\left(\xi_{1}^{4}+4 \xi_{1}^{2} \beta_{p 1}^{2}+2 \beta_{p 1}^{4}\right)\right. \\
& \left.-\frac{d_{1}^{2} \omega^{2}}{3 V_{s 1}^{2}} \beta_{p 1}^{2}\right] \\
& b_{71}=-2 \xi_{1} \beta_{p 1}\left(b_{y}^{(1)}-c_{1} \beta_{p 1} i\right) \mu_{1}, \\
& b_{81}=2 \mu_{1} \zeta_{1}\left(\sigma_{p 1}^{2}+\beta_{p 1}^{2}\right)\left(b_{y}^{(1)}-c_{1} \beta_{s 1} i\right), \\
& c_{11}=\beta_{s 1}, \quad c_{21}=\zeta_{1}, \quad c_{31}=\beta_{s 1}^{2}, \quad c_{41}=\zeta_{1} \beta_{s 1}, \\
& c_{51}=-\mu_{1}\left[\left(\zeta_{1}^{2}-\beta_{s 1}^{2}-2 b_{y}^{(1)} \zeta_{1}^{2} \beta_{s 1} i\right)-c_{1}\left(\beta_{s 1}^{4}-\zeta_{1}^{4}+2 \zeta_{1}^{2} \beta_{s}^{2}\right)\right. \\
& \left.+\frac{d_{1}^{2} \omega^{2}}{3 V_{s 1}^{2}} \beta_{s 1}^{2}\right] \\
& c_{61}=\mu_{1}\left[2 \zeta_{1} \beta_{s 1}+b_{y}^{(1)} \zeta_{1}\left(\zeta_{1}^{2}-\beta_{s 1}^{2}\right) i+c_{1} \zeta_{1} \beta_{s 1}\left(\sigma_{s 1}^{2}+2 \zeta_{1}^{2}\right)\right. \\
& \left.-\frac{d_{1}^{2} \omega^{2}}{3 V_{s 1}^{2}} \zeta_{1} \beta_{s 1}\right] \\
& c_{71}=-\left(\zeta_{1}^{2}-\beta_{s 1}^{2}\right)\left(b_{y}^{(1)}-c_{1} \beta_{s 1} i\right) \mu_{1}, \\
& c_{81}=2 \mu_{1} \zeta_{1} \beta_{s 1}\left(b_{y}^{(1)}-c_{1} \beta_{s 1} i\right) \text {, }
\end{aligned}
$$

\section{References}

Cai, Bei, Wei, Peijun, 2013. Surface/interface effects on dispersion relations of 2D phononic crystal with parallel nanofibers. Acta Mech. 224 (11), 2749-2758.

Duan, H.L., et al., 2005. Size-dependent effective elastic constants of solids containing nano-inhomogeneities with interface stress. J. Mech. Phys. Solids 53 (7), 1574-1596.

Eringen, A.C., 1966. Linear theory of micropolar elasticity. J. Math. Mech. 15, 909-924.
Eringen, A.C., 1990. Theory of thermo-microstretch elastic solids. Int. J. Eng. Sci. 28, 1291-1301.

Eringen, A.C., 2001. Nonlocal Continuum Field Theories. Springer-Verlag, Berlin, Heidelberg.

Eringen, A.C., 1964. Mechanics of micromorphic materials. In: Gortler, H. (Ed.), Proc. XI, Int. Congress of Applied Mechanics. Springer Verlag, New York.

Exadaktylos, G., 1999. Some basic half-plane problems of the cohesive elasticity theory with surface energy. Acta Mech. 133, 175-198.

Georgiadis, H.G., Vardoulakis, I., Lykotrafitis, G., 2000. Torsional surface waves in a gradient-elastic half-space. Wave Motion 31, 333-348.

Gourgiotis, P.A., Georgiadis, H.G., Neocleous, I., 2013. On the reflection of waves in half-spaces of microstructured materials governed by dipolar gradient elasticity. Wave Motion 50, 437-455.

Graff, K.F., Pao, Yih-Hsing, 1967. The effects of couple-stresses on the propagation and reflection of plane waves in an elastic half-space. J. Sound. Vib. 6 (2), 217-229.

Gurtin, M.E., Murdoch, A.I., 1975. A continuum theory of elastic material surfaces. Arch. Rat. Mech. Anal. 57, 291-323.

Hasheminejad, S.M., Avazmohammadi, R., 2009. Size-dependent effective dynamic properties of unidirectional nanocomposites with interface energy effects. Compos. Sci. Technol. 69, 2538-2546.

dell'Isola, F., Madeo, A., Placidi, L., 2012. Linear plane wave propagation and normal transmission and reflection at discontinuity surfaces in second gradient 3D Continua. Z. Angew. Math. Mech. (ZAMM) 92, 52-71.

Liu, W., Chen, J., Liu, Y., Su, X., 2012. Effect of interface/surface stress on the elastic wave band structure of two-dimensional phononic crystals. Phys. Lett. A 376, 605-609.

Miller, R.E., Shenoy, V.B., 2000. Size-dependent elastic properties of nanosized structural elements. Nanotechnology 11 (3), 139-147.

Mindlin, R.D., Tiersten, H.F., 1962. Effects of couple stress in linear elasticity. Arch. Ration. Mech. Anal. 11, 415-448.

Ogden, R.W., Steigmann, D.J., 2002. Plane strain dynamics of elastic solids with intrinsic boundary elasticity, with application to surface wave propagation. J. Mech. Phys. Solids 50, 1869-1896.

Parfitt, V.R., Eringen, A.C., 1969. Reflection of plane waves from the flat boundary of a micropolar half-space. J. Acoust. Soc. Am. 45, 1258-1272.

Placidi, L., Rosi, G., Giorgio, I., Madeo, A., 2013. Reflection and transmission of plane waves at surfaces carrying material properties and embedded in second gradient materials. Math. Mech. Solids 19, 555-578.

Qiang, F.W., Wei, P.J., 2013. Propagation of elastic wave in nanoporous material with distributed cylindrical nanoholes. Sci. China Phys Mech. Astron. 56, 1542-1550.

Qiang, F.W., Wei, P.J., Li, L., 2012. The effective propagation constants of SH wave in composites reinforced by dispersive parallel nanofibers. Sci. China Phys. Mech. Astron. 55 (7), 1172-1177.

Shenoy, V.B., 2005. Atomistic calculations of elastic properties of metallic fcc crystal surfaces. Phys. Rev. B 71 (1-14), 094104.

Tomar, S.K., Garg, Monika, 2005. Reflection and transmission of waves from a plane interface between two microstretch solid half-spaces. Int. J. Eng. Sci. 43, $139-169$.

Tomar, S.K., Gogna, M.L., 1995. Reflection and refraction of longitudinal wave at an interface between two micropolar elastic solids at welded Contact. J. Acoust. Soc. Am. 97 (2), 822-830.

Toupin, R.A., 1962. Elastic materials with couple stresses. Arch. Ration. Mech. Anal. 11, 385-414.

Tsepoura, K.G., Papargyri, S., Polyzos, D., 2002. A boundary element method for solving 3D static gradient elastic problems with surface energy. Comput. Mech. $29,361-381$.

Vardoulakis, I., Georgiadis, H.G., 1997. SH surface waves in a homogeneous gradient elastic half-space with surface energy. J. Elast. 47, 147-165.

Wang, G.F., 2007. Diffraction of plane compressional wave by a nanosized spherical cavity with surface effects. Appl. Phys. Lett. 90, 211907.

Wang, G.F., 2008. Diffraction of shear waves by a nanosized spherical cavity. Appl. Phys. Lett. 103, 053519.

Yerofeyev, V.I., Sheshenina, O.A., 2005. waves in gradient elastic medium with surface energy. J. Appl. Math. Mech. 69, 57-59. 